\title{
SPECIALIZATIONS OF ONE-PARAMETER FAMILIES OF POLYNOMIALS
}

\author{
FARSHID HAJIR AND SIMAN WONG
}

\begin{abstract}
Let $K$ be a number field, and let $\lambda(x, t) \in K[x, t]$ be irreducible over $K(t)$. Using algebraic geometry and group theory, we study the set of $\alpha \in K$ for which the specialized polynomial $\lambda(x, \alpha)$ is $K$-reducible. We apply this to show that for any fixed $n \geq 10$ and for any number field $K$, all but finitely many $K$-specializations of the degree $n$ generalized Laguerre polynomial $L_{n}^{(t)}(x)$ are $K$-irreducible and have Galois group $S_{n}$. In conjunction with the theory of complex multiplication, we also show that for any $K$ and for any $n \geq 53$, all but finitely many of the $K$-specializations of the modular equation $\Phi_{n}(x, t)$ are $K$-irreducible and have Galois group containing $P S L_{2}(\mathbf{Z} / n)$.
\end{abstract}

\section{Contents}

\begin{tabular}{|c|c|}
\hline \multicolumn{2}{|l|}{ 1. Introduction } \\
\hline 2. Rational specializations & 4 \\
\hline 3. Modular equations & 5 \\
\hline 4. A Riemann-Hurwitz estimate & 7 \\
\hline 5. Specializations of $S_{n}$-covers & 1 . \\
\hline 5.1. Distinguished subfields in $S_{n}$-extensions & 11 \\
\hline 5.2. Interpretation in terms of fiber products & $1:$ \\
\hline 6. Generalized Laguerre Polvnomials & 1 \\
\hline 6.1. The singular locus of $L_{n}^{(t)}(x)$ & 16 \\
\hline 6.2. Genus of maximal subgroups & 18 \\
\hline 6.3. Proof of Theorem [1] & \\
\hline 7. Simple covers & \\
\hline References & \\
\hline
\end{tabular}

\section{INTRODUCTION}

Let $K$ be a number field. Consider a polynomial $\lambda(x, t) \in K[x, t]$ which is non-constant in each of $x$ and $t$; it can be viewed as a one-parameter family of $K$-polynomials in $x$. If $\lambda$ is irreducible in $K[x, t]$, the Hilbert irreducibility theorem furnishes infinitely many $\alpha \in K$ for which $\lambda(x, \alpha)$ is $K$-irreducible. It is then natural to study the set of $\alpha \in K$ with reducible specialization. These exceptional sets are thin sets [29, §9.6], and the example $x^{n}-t$ shows that they can be infinite. Using techniques from diophantine analysis, Fried [10] bounded

1991 Mathematics Subject Classification. Primary 12H25; Secondary 11C08, 11G15,11R09, 14H25, 33C45.

Key words and phrases. Branched cover, complex multiplication, Hilbert irreducibility, modular equation, orthogonal polynomial, rational point, Riemann-Hurwitz formula, simple cover, specialization.

Hajir's research is supported in part by NSF Grant No. 0226869. 
the number of exceptional specializations of bounded height. Exceptional sets for concrete families have also been examined; for example the irreducibility and Galois group of the Generalized Laguerre polynomial

$$
L_{n}^{(t)}(x)=\sum_{j=0}^{n}(-x)^{j}\left(\begin{array}{l}
n \\
j
\end{array}\right) \prod_{k=j+1}^{n}(t+k) .
$$

for various rational values of the parameter $t$ were studied by Schur (26, 27]); more recently, Feit 9] used them to solve the inverse Galois problem over $\mathbf{Q}$ for certain double covers of the alternating group $A_{n}$. See also [14, [15], [28], [16], for other related results. Note that in the papers just cited, the focus is primarily on a related, but different, question from the one we began with, namely that of irreducibility and Galois properties of $L_{n}^{\left(\alpha_{n}\right)}(x)$ for suitable sequences $\left\{\alpha_{n}\right\}_{n}$. For example, the case $\alpha_{n}=-1-n$ corresponds to the truncated exponential polynomial studied by Schur [26]. For the latter type of question, the $p$-adic Newton polygon is a powerful tool. For example, in Filaseta-Lam [13] it is shown that if we fix $\alpha \in \mathbf{Q}-\mathbf{Z}_{<0}$, then $L_{n}^{(\alpha)}(x)$ is $\mathbf{Q}$-irreducible for $n$ sufficiently large, while in FilasetaTrifonov [12], Grosswald's conjecture, to the effect that $L_{n}^{(-1-2 n)}(x)$ (i.e. the $n$-th degree Bessel polynomial) is Q-irreducible for every $n$, is proved. The Newton Polygon approach, however, does not appear to be well-suited to the problem under consideration here, namely that of studying exceptional specializations of $L_{n}^{(t)}(x)$ for $n$ fixed.

In this paper we investigate the exceptional set of a given $\lambda(x, t)$ from the algebro-geometric and group-theoretic points of view. First, note that $\lambda(x, t)$ defines a 1-dimensional subvariety $X_{\lambda} \subset \mathbf{P}_{K}^{2}$. To say that the specialization of $\lambda$ at $t=\alpha$ has a $K$-rational root is to say that the fiber above $\alpha$ of the projection-to- $t$ map has a $K$-rational point. Say $X_{\lambda}$ is in fact absolutely irreducible; then, by Faltings, at most finitely many $K$-specializations of $\lambda$ have a $K$-rational root if $X_{\lambda}$ has genus $\geq 2$. More generally, a result of Müller 23 leads to an irreducibility criterion for specializations in terms of the genus of intermediate subfields of $K^{\prime} / K(t)$ where $K^{\prime}$ is the Galois closure of $\lambda(x, t)$ over the function field $K(t)$ (cf. also the related results of Dèbes and Fried [6]). In sections 2 [5, we develop and refine tools for applying this criterion. In section 6, we apply these to study $L_{n}^{(t)}(x)$. The recursive properties of $L_{n}^{(t)}(x)$ allow us to analyze the geometry of the corresponding curve as well as the ramification behavior of the projection-to- $t$ map. By utilizing, in addition, information about maximal subgroups of the symmetric group $S_{n}$, we obtain the following result.

Theorem 1. Let $K$ be a number field.

(a) Fix $n \geq 5$. Then for all but finitely many $\alpha \in K, L_{n}^{(\alpha)}(x)$ is K-irreducible and its Galois group (over $K$ ) contains $A_{n}$. For fixed $n \geq 10$, this Galois group is exactly $S_{n}$ except for finitely many $\alpha \in K$.

(b) Let $R$ be a finitely generated subring of $K$. If $n \geq 6$, then for all but finitely many $\alpha \in R$, the Galois group over $K$ of $L_{n}^{(\alpha)}(x)$ is exactly $S_{n}$.

Remark 1. Note that Theorem [1 is optimal in two ways. First, for $6 \leq n \leq 9$, the set of $\alpha \in K$ for which the discriminant of $L_{n}^{(\alpha)}(x)$ is a square in $K$ turns out to be parameterized by a curve of geometric genus one, so for suitable $K$ there are infinitely many specializations 
with even Galois group. And when $n=5$, the square discriminants are parameterized by a curve of geometric genus zero, so there are fields $K$ and finitely generated subrings $R$ of $K$ over which there are infinitely many even specializations. Second, $L_{4}^{(t)}(x)=0$ is a model (cf. [16]) of the elliptic curve $384 H 2$ in Cremona's table. This curve has Mordell-Weil rank 1 over $\mathbf{Q}$, so over any number field $K$ there are infinitely many $\alpha \in K$ for which $L_{4}^{(\alpha)}(x)$ has a $K$-rational linear factor. However, the exceptional set in Theorem 1 is captured by rational points on curves of high geometric genus, so it would be difficult to make the Theorem effective.

Before we develop the tools necessary for proving Theorem refthm:irr, we illustrate the use of Müller's criterion by applying it to another well-studied polynomial, namely the modular polynomial $\Phi_{n}(x, j)$. This monic $\mathbf{Z}$-polynomial plays a central role in the theory of elliptic curves; it is determined up to a scalar multiple by the property that two elliptic curves over $\mathbf{C}$ with $j$-invariants $j_{1}, j_{2}$ are related by a cyclic $n$-isogeny if and only if $\Phi_{n}\left(j_{1}, j_{2}\right)=0$. It is irreducible over $\mathbf{C}(j)$, and its Galois group over $\mathbf{Q}(j)$ is $P G L_{2}(\mathbf{Z} / n)$.

For any integer $n>1$ and any prime $p$, define

$$
\mathbf{Q}_{p, n}= \begin{cases}\text { unique quadratic extension of } \mathbf{Q} \text { of conductor } p & \text { if } p>2 \text { and } p \mid n, \\ \text { unique biquadratic extension of } \mathbf{Q} \text { of conductor } 8 & \text { if } p=2 \text { and } 8 \mid n \\ \text { unique quadratic extension of } \mathbf{Q} \text { of conductor } 4 & \text { if } p=2 \text { and } 4|| n \\ \mathbf{Q} & \text { otherwise }\end{cases}
$$

For any number field $K$ and any $n>1$, denote by $\tilde{K}_{n}$ the compositum of $K$ with all $\mathbf{Q}_{p, n}$ as $p$ runs over the prime divisors of $n$; note that this is a finite extension of $K$.

Theorem 2. Let $n \geq 53$, and let $K$ be a number field. Then for all but finitely many $\alpha \in \tilde{K}_{n}$, $\Phi_{n}(x, \alpha)$ is $K$-irreducible, and its Galois group over $\tilde{K}_{n}$ is $P S L_{2}(\mathbf{Z} / n)$. If $n$ is a prime then it suffices to take $n \geq 23$.

Remark 2. Theorem 2 is close to optimal in the $n$-aspect; cf. Remark 3 . However, as in the discussion following Theorem 1, it would be difficult to make Theorem 2 effective.

We will describe our strategy via Müller's criterion in section 2, after we establish some notation. To apply this criterion to specializations of $\Phi_{n}$, in section 3 we investigate the algebraic closure of $\mathbf{Q}$ in the function field defined by $\Phi_{n}$, and we study the genus of Riemann surfaces defined by congruence subgroups. In sections 4 and 5 , we develop the technical tools needed for carrying out the strategy outlined in section 2. In section 6] we implement this plan for the Generalized Laguerre Polynomial after first establishing several geometric properties of the projective plane curve $\mathcal{L}_{n}$ defined by $L_{n}^{(t)}(x)=0$. Specifically, let $\iota_{n}$ : $\mathcal{L}_{n} \rightarrow \mathbf{P}_{K}^{1}$ be the branched cover defined by the projection-to- $t$ map. Then

(i) $K$ is algebraically closed in the splitting field of $L_{n}^{(t)}$ over $K(t)$;

(ii) the (geometric) Galois group of $\iota_{n}$ is $S_{n}$;

(iii) $L_{n}^{(t)}(x)$, as a polynomial in $x$, has discriminant which is non-constant in $t$;

(iv) $\mathcal{L}_{n}$ has no affine singular points, and

(v) $\iota_{n}$ has several "simple" branch points of index close to $n$. 
In (v), a simple branch point of index $e$ is one whose fiber consists of a number (possibly 0) of multiplicity one points together with a single ramified point (of multiplicity $e$ ). The cover defined by the degree $n$ Generalized Laguerre Polynomial has one simple branch point of every index between 2 and $n$ : we use the four of highest index, which suffices in our analysis for all $n \geq 6$. As the calculations in section [6] will show, the proof of Theorem 1 extends readily to other one-parameter families of polynomials satisfying properties (i)-(v) (as long as their degree is large with respect to the precise form taken by condition $(\mathrm{v}))$. On the other hand, given an arbitrary $\lambda(x, t)$ which is irreducible over $K(t)$, in general we cannot expect all but finitely many of its $K$-specializations to be $K$-irreducible, let alone having the same Galois group as $\lambda(x, t)$ over $K(t)$ - the subvariety $X_{\lambda}$ mentioned just before the statement of Theorem [1 could, for example, have genus $\leq 1$. In section 7 we will analyze this situation further in the case of "simple branched covers," i.e. where all the branch points are simple of index 2 .

\section{RATIONAL SPECIALIZATIONS}

We first establish some notation and hypotheses which will be maintained throughout. Let $K$ be a field of characteristic 0 , finitely generated over Q. Fix an algebraic closure $\bar{K}$ of $K$. Denote by $K_{0}$ the function field $K(t)$. Fix $\lambda(x, t) \in K[x, t]$ so that $\lambda$ has degree $n>0$ in $x$ and is irreducible over $K_{0}$. Then $K_{1}:=K[x] /(\lambda(x, t))$ is a degree $n$ extension of $K_{0}$. Let $K^{\prime} / K_{0}$ be a Galois closure of $K_{1} / K_{0}$, and write $G_{\lambda}=\operatorname{Gal}\left(K^{\prime} / K_{0}\right)$. By [29, p. 123], the Galois group of $\lambda(x, \alpha)$ over $K$ is a subgroup of $G_{\lambda}$ for any $\alpha \in K$, and by [29. Prop. 9.2], there are infinitely many $\beta_{0} \in K$ for which this Galois group is exactly $G_{\lambda}$.

From now on, suppose that

(i) $K$ is algebraically closed in $K^{\prime} / K_{0}$.

Then [31, Remark II.2.5] implies that every intermediate subfield $E$ of $K^{\prime} / K_{0}$ is the function field of a smooth projective curve $X_{E}$ over $K$, and if $E \subset E^{\prime}$ are two such subfields, then there exists a $K$-morphism $X_{E^{\prime}} \rightarrow X_{E}$ of degree $\left[E^{\prime}: E\right]$. We write $g\left(X_{E}\right)$ for the genus of $X_{E}$. By Galois theory, intermediate fields $E$ of $K^{\prime} / K_{0}$ are in bijective correspondence with subgroups $\mathcal{E}=\operatorname{Gal}\left(K^{\prime} / E\right)$ of $G_{\lambda}$. To simplify the exposition, we abbreviate the phrase 'all but finitely many $\alpha \in K^{\prime}$ by $\alpha \in \in_{\text {af }} K$.

Proposition 1. Let $K^{\prime} / K_{0}$ be as above, and consider a polynomial $f \in K[x, t]$ which is irreducible over $K_{0}$ but splits completely into linear factors over $K^{\prime}$. Suppose for every intermediate subfield $E$ of $K^{\prime} / K_{0}$ such that $f$ is reducible over $E$, we have $g\left(X_{E}\right)>1$. Then $f(x, \alpha)$ is $K$-irreducible for $\alpha \in_{a f} K$.

Proof. This is probably well-known to the expert; for a convenient reference see Müller [23, Prop. 4.20]. A method of proof is also indicated in 5.2 .

For any $\alpha \in K$, the Galois group of $\lambda(x, \alpha)$ over $K$ is a subgroup of $G_{\lambda}$, and we are interested in finding conditions on $\alpha$ under which $\lambda(x, \alpha)$ is not only $K$-irreducible, but also has Galois group coinciding with the full $G_{\lambda}$. Here is our strategy: suppose the splitting field of some "test-polynomial" $f(x, t) \in K[x, t]$ is contained in $K^{\prime}$; then the splitting field of $f(x, \alpha)$ over $K$ is contained in that of $\lambda(x, \alpha)$. So if $f(x, \alpha)$ is $K$-irreducible, then the 
degree of the splitting field of $\lambda(x, \alpha)$ over $K$ would be divisible by the degree of $f(x, \alpha)$. By running through an appropriate collection of $f$ (e.g. the polynomials $\Lambda_{j}$ introduced in 51), we can then hope to show that $\# G_{\lambda}$ divides the degree of the splitting field of $\lambda(x, \alpha)$ over $K$, whence the Galois group of $\lambda(x, \alpha)$ over $K$ must be $G_{\lambda}$. To study the irreducibility of the specializations $f(x, \alpha)$ we use Proposition 1, which reduces the problem to estimating the genus of $X_{E}$ as we run through intermediate subfields $E$ of $K^{\prime} / K_{0}$.

\section{Modular Equations}

By [20, p. 55], the modular polynomial $\Phi_{n}(x, j) \in \mathbf{Z}[x, j]$ is irreducible over $\mathbf{C}(j)$. We now apply the strategy developed in the last section to study specializations of $\Phi_{n}$. Denote by $L_{n}$ the splitting field of $\Phi_{n}$ over $\mathbf{Q}(t)$. Recall the definition of $\mathbf{Q}_{p, n}$ and $\tilde{K}_{n}$ immediately preceding the statement of Theorem 2 .

Lemma 1. The algebraic closure of $\mathbf{Q}$ in $L_{n} / \mathbf{Q}(t)$ is $\tilde{\mathbf{Q}}_{n}$.

Proof. As a coarse moduli scheme, the open modular curve $Y_{0}(n)$ classifies isomorphism classes $\left(E \rightarrow E^{\prime}\right)$ of pairs of elliptic curves related via a cyclic $n$-isogeny. Over the complex numbers, such a pair is completely determined by the $j$-invariants of $E$ and $E^{\prime}$. Thus the complex points of $Y_{0}(n)$ are canonically identified with the complex points of the affine plane curve defined by $\Phi_{n}(x, j)=0$. Under this identification, the projection-to- $j$ map from this complex plane curve corresponds precisely to the branched cover $\pi_{0}(n): Y_{0}(n) \rightarrow Y_{0}(1)$ coming from the inclusion $\Gamma_{0}(n) \subset S L_{2}(\mathbf{Z})$. The smallest regular branched cover containing $\pi_{0}(n)$ is then the cover $\pi(n): Y(n) \rightarrow Y(1)=Y_{0}(1)$ corresponding to the inclusion $\Gamma(n) \subset S L_{2}(\mathbf{Z})$. In particular, the deck transformation group of $\pi(n)$ is

$$
P S L_{2}(\mathbf{Z}) /(\Gamma(n) / \pm I) \simeq P S L_{2}(\mathbf{Z} / n) .
$$

It follows that the geometric Galois group of $\Phi_{n}$ is $P S L_{2}(\mathbf{Z} / n)$. But Macbeath [22] showed that $\operatorname{Gal}\left(L_{n} / \mathbf{Q}(t)\right) \simeq P G L_{2}(\mathbf{Z} / n)$, so the algebraic closure of $\mathbf{Q}$ in $L_{n} / \mathbf{Q}(t)$ is the compositum of $\mathbf{Q}(t)$ with a Galois extension $L(n) / \mathbf{Q}$ with Galois group

$$
\begin{aligned}
P G L_{2}(\mathbf{Z} / n) / P S L_{2}(\mathbf{Z} / n) & \simeq \prod_{p \mid n} P G L_{2}\left(\mathbf{Z} / p^{e_{p}}\right) / P S L_{2}\left(\mathbf{Z} / p^{e_{p}}\right) \quad \text { where } p^{e_{p}} \| n \\
& \simeq \prod_{\substack{p \mid n \\
p>2}}(\mathbf{Z} / 2) \times\left\{\begin{array}{ll}
\mathbf{Z} / 2 \times \mathbf{Z} / 2 & \text { if } 8 \mid n \\
\mathbf{Z} / 2 & \text { if } 4|| n \\
\{1\} & \text { otherwise }
\end{array}\right\} .
\end{aligned}
$$

If $m \mid n$ then $L_{m} \subset L_{n}$, hence $L(m) \subset L(n)$, so to prove the Lemma we are reduced to showing that for any prime power $p^{e}>1$,

$$
L\left(p^{e}\right)=\mathbf{Q}_{p, p^{e}}
$$

For any $\alpha \in \mathbf{Q}$ and any $n>1$, the splitting field of $\Phi_{n}(x, \alpha)$ over $\mathbf{Q}$ also contains $L(n)$. Take $\alpha \in \mathbf{Q}$ to be one of the thirteen $j$-invariants over $\mathbf{Q}$ corresponding to CM elliptic curves over $\mathbf{Q}$, say $\alpha=j(\tau)$. Denote by $k_{\alpha} / \mathbf{Q}$ the corresponding complex quadratic field. By the 'First Main Theorem' of complex multiplication [4, Thm. 11.1], $k_{\alpha}(j(n \tau))$ is the ring class field of $k_{\alpha}$ of conductor $n$, hence $L(n) \subset k_{\alpha}(j(n \tau))$. In particular, $L(n) / \mathbf{Q}$ is unramified 
outside of the prime divisors of $n$ and of the discriminant of $k_{\alpha} / \mathbf{Q}$. If $j\left(\tau^{\prime}\right)=\alpha^{\prime} \in \mathbf{Q}$ is another CM $j$-invariant over $\mathbf{Q}$, then $L(n) \subset k_{\alpha}(j(n \tau)) \cap k_{\alpha}^{\prime}\left(j\left(n \tau^{\prime}\right)\right)$. We may choose $\alpha^{\prime}$ so that $k_{\alpha}$ and $k_{\alpha}^{\prime}$ have coprime discriminants, whereby $L\left(p^{e}\right) / \mathbf{Q}$ is unramified outside $p$. On the other hand, (2) says that $L\left(p^{e}\right) / \mathbf{Q}$ is quadratic if $p>2$ or $p^{e}=4$, and that it is biquadratic if $8 \mid p^{e}$. Recalling the definition of $\mathbf{Q}_{p, n}$, we get (3) except when $p^{e}=4$. To treat this remaining case we actually need to determine these ring class fields.

Set $\omega=\frac{1+\sqrt{-7}}{2}$, and take $\alpha=j(\omega) \in \mathbf{Q}$, so $k_{\alpha}=\mathbf{Q}(\omega)$. The conductor of the extension $k_{\alpha}(\sqrt{-1}) / k_{\alpha}$ clearly divides $4 \mathbf{Z}[\omega]$. On the other hand, by [4, Thm. 7.24] the ring class field of $k_{\alpha}$ of conductor $4 \mathbf{Z}[\omega]$ is a quadratic extension of $k_{\alpha}$, so this ring class field is precisely $k_{\alpha}(\sqrt{-1})$. Recalling (2) , we see that $L(4) / \mathbf{Q}$ is a quadratic extension in $\mathbf{Q}(\omega, \sqrt{-1})$ unramified outside 2 , and (3) follows for $p^{e}=4$.

Rademacher conjectured that there are only finitely many congruence subgroups with corresponding modular curve of genus zero (cf. [19]). Dennin [18] proved the stronger result that for any integer $g$, there are at most finitely many $n$ for which $P S L_{2}(\mathbf{Z} / n)$ contains a subgroup of genus $\leq g$. Cummins and Pauli [5] recently tabulated all such subgroups for $g \leq 24$, from which we deduce the following result.

Lemma 2 (Cummins-Pauli). If $n \geq 53$, then every proper subgroup of $P S L_{2}(\mathbf{Z} / n)$ has genus $\geq 2$. If $n$ is a prime, the same conclusion holds for $n \geq 23$.

Proof of Theorem Q. Thanks to Lemma 1, the discussion in section 2 is applicable to $\Phi_{n}$ over $\tilde{K}_{n}$ for any number field $K$.

Let $\pi_{n}$ be a primitive element for the extension $\tilde{K}_{n} L_{n}(t) / \tilde{K}_{n}(t)$, and let $f_{n}(x, t)$ be the minimal polynomial of $\pi_{n}$ over $\tilde{K}_{n}(t)$. Then $f_{n}$ is irreducible over $\tilde{K}_{n}(t)$, by construction. So if $n$ is as in Lemma 2. then Proposition 1 and this Lemma together imply that for $\alpha \in_{\text {af }} \tilde{K}_{n}$, the specializations of $f_{n}$ and of $\Phi_{n}$ at $t=\alpha$ are both $\tilde{K}_{n}$-irreducible. If we write $F_{n}(\alpha)$ for the splitting field of $\Phi_{n}(x, \alpha)$ over $\tilde{K}_{n}$, then that means $\left[F_{n}(\alpha): \tilde{K}_{n}\right]$ is divisible by $\operatorname{deg} f_{n}=\left[\tilde{K}_{n} L_{n}(t): \tilde{K}_{n}(t)\right]=\# P S L_{2}(\mathbf{Z} / n)$, and Theorem 2 follows.

Remark 3 . The fact that every non-trivial intermediate subfield of $\tilde{K}_{n} L_{n}(t) / \tilde{K}_{n}(t)$ has genus $\geq 2$ for $n \geq 53$ significantly simplifies our search for the 'test polynomial' $f$ in Proposition 1. The modular curve $X_{0}(n)$ has genus $\leq 1$ for $n \leq 21$ and for $n \in\{24,25,27,32,36,49\}$, so by the discussion immediately preceding Theorem 11 for these $n$ the modular equation has infinitely many reducible specializations over suitable $K$. To analyze the remaining values of $n \leq 52$ we could search for test polynomials $f$ which remain irreducible over intermediate subfields of genus $\leq 1$. We will not pursue this issue here, but in section [5 we will study the same problem for specializations of $S_{n}$-extensions by using a family of $[(n-1) / 2]$ test polynomials $\Lambda_{j}(x, t)$. 


\section{A Riemann-Hurwitz estimate}

We now return to the general setup in section 2. To apply Proposition 1, we need to be able to estimate the genus of certain intermediate subfields of $K^{\prime} / K_{0}$. To do that we will apply the Riemann-Hurwitz formula to the cover $\xi_{E}: X_{E} \rightarrow \mathbf{P}_{K}^{1}$ corresponding to the field inclusion $K_{0} \subset E$. Since we do not have any explicit model for $X_{E}$, we will take an algebraic approach. Thanks to hypothesis (i) in section 2, in order to determine the ramification of the geometric cover $X^{\prime} \rightarrow \mathbf{P}_{K}^{1}$ it suffices to determine the algebraic ramification behavior of integral extensions of Dedekind domains corresponding to this geometric cover.

Denote by $B_{\lambda} \subset \mathbf{P}_{K}^{1}$ the branch locus of the projection-to- $t$ map for $\lambda$. Then $\xi_{E}$ is unramified outside $B_{\lambda}$. Fix affine open sets on $X_{E}$ and $X^{\prime}$ which contain every fiber of $\xi_{E}$ and $X^{\prime} \rightarrow \mathbf{P}_{K}^{1}$ above $B_{\lambda}$, and denote by $\mathcal{O}_{E}$ and $\mathcal{O}^{\prime}$ their respective affine coordinate rings. Write $\mathcal{O}_{0}$ for the affine coordinate ring of the affine line in $\mathbf{P}_{K}^{1}$. Let $\mathfrak{m}_{\nu}$ (or just $\mathfrak{m}$ if $\nu$ is fixed) be the maximal ideal in $\mathcal{O}_{0}$ corresponding to a given $\nu \in B_{\lambda}$. We let $e_{\nu}=e(\mathfrak{M} / \mathfrak{m})$ be the ramification index of $\mathfrak{M}$ in the Galois cover $K^{\prime} / K_{0}$, where $\mathfrak{M}$ is an arbitrary prime of $\mathcal{O}^{\prime}$ dividing $\mathfrak{m} \mathcal{O}^{\prime}$.

Definition 1. (a) For a positive integer $\delta$ and a branch point $\nu \in B_{\lambda}$ corresponding to an ideal $\mathfrak{m} \in \mathcal{O}_{0}$, let

$$
c_{\delta}(\nu)=c_{\delta}(\mathfrak{m})=\sum_{\substack{\mathfrak{n} \mid \mathfrak{m} \mathcal{O}_{E} \\ e(\mathfrak{n} / \mathfrak{m})=\delta}} f(\mathfrak{n} / \mathfrak{m})
$$

be the sum of the residual degrees of distinct $\mathcal{O}_{E}$-primes $\mathfrak{n}$ of ramification index $\delta$ over $\mathfrak{m}$.

(b) For $\nu \in B_{\lambda}$ corresponding to an ideal $\mathfrak{m} \in \mathcal{O}_{0}$, let

$$
\Delta(\nu)=\Delta(\mathfrak{m})=\sum_{\mathfrak{n} \mid \mathfrak{m} \mathcal{O}_{E}}(e(\mathfrak{n} / \mathfrak{m})-1) f(\mathfrak{n} / \mathfrak{m})
$$

be the $\nu$-component of the discriminant of $E / K_{0}$.

(c) For an integer $e>1$, let $d(e)$ be the least prime divisor of $e$.

Lemma 3. With the notation and hypotheses as in section 2, if $E$ is an intermediate field of $K^{\prime} / K_{0}$ corresponding to a subgroup $\mathcal{E}=\operatorname{Gal}\left(K^{\prime} / E\right)$ of $G_{\lambda}=\operatorname{Gal}\left(K^{\prime} / K_{0}\right)$, and $V$ is any subset of $B_{\lambda}$, then

$$
g\left(X_{E}\right) \geq 1+\frac{[G: \mathcal{E}]}{2}\left(-2+\sum_{\nu \in V}\left(1-\frac{1}{d\left(e_{\nu}\right)}\right)\right)-\frac{1}{2} \sum_{\nu \in V} c_{1}(\nu)\left(1-\frac{1}{d\left(e_{\nu}\right)}\right) .
$$

Proof. First, note that

$$
\sum_{1 \leq \delta \mid e_{\nu}} c_{\delta}(\nu) \delta=\left[E: K_{0}\right]=\left[G_{\lambda}: \mathcal{E}\right]
$$


For each $\nu \in B_{\lambda}$, we have from Definition 1,

$$
\begin{aligned}
\Delta(\nu) & =\sum_{1 \leq \delta \mid e_{\nu}} c_{\delta}(\nu)(\delta-1) \\
& =\sum_{1<\delta \mid e_{\nu}} c_{\delta}(\nu)\left(1-\frac{1}{\delta}\right) \delta \\
& \geq\left(1-\frac{1}{d\left(e_{\nu}\right)}\right) \sum_{1<\delta \mid e_{\nu}} c_{\delta}(\nu) \delta \\
& \geq\left(1-\frac{1}{d\left(e_{\nu}\right)}\right) \sum_{1 \leq \delta \mid e_{\nu}} c_{\delta}(\nu) \delta-\left(1-\frac{1}{d\left(e_{\nu}\right)}\right) c_{1}(\nu) \\
& \geq\left[G_{\lambda}: \mathcal{E}\right]\left(1-\frac{1}{d\left(e_{\nu}\right)}\right)-c_{1}(\nu)\left(1-\frac{1}{d\left(e_{\nu}\right)}\right) \quad \text { by (15). }
\end{aligned}
$$

By Riemann-Hurwitz for $E / K_{0}$, [24, Theorem 7.16], we have

$$
g\left(X_{E}\right)-1=\left[E: K_{0}\right](0-1)+\frac{1}{2} \sum_{\nu \in B_{\lambda}} \Delta(\nu) .
$$

Since $\Delta(\nu)>0$, we have, for any subset $V \subseteq B_{\lambda}$,

$$
\begin{aligned}
g\left(X_{E}\right) & \geq 1-\left[G_{\lambda}: \mathcal{E}\right]+\frac{1}{2} \sum_{\nu \in V} \Delta_{\nu} \\
& \geq 1+\frac{\left[G_{\lambda}: \mathcal{E}\right]}{2}\left(-2+\sum_{\nu \in V}\left(1-\frac{1}{d\left(e_{\nu}\right)}\right)\right)-\frac{1}{2} \sum_{\nu \in V} c_{1}(\nu)\left(1-\frac{1}{d\left(e_{\nu}\right)}\right) \text { by (6) } .
\end{aligned}
$$

Remark 4. Note that the bound (4) is useful only when $c_{1}(\nu)$ is fairly small for all $\nu \in V$, so in using (41), it's often useful to take $V$ to be a proper subset of $B_{\lambda}$. Moreover, the inequality (44) is in fact strict if $V$ is a proper subset of $B_{\lambda}$ since $\Delta(\nu)>0$ for $\nu \in V$.

In view of Proposition 1, our task will be to show that the right hand side of (4) is $>1$ when a given $f(x, t) \in K[x, t]$ is reducible over $E$. For our application to Generalized Laguerre Polynomials, this will be easy to arrange by taking $V$ to be an appropriately small subset of $B_{\lambda}$.

We now turn to the task of bounding $c_{1}=c_{1}(\nu)$ from above, where, for the remainder of this section, $\nu \in B_{\lambda}$ is a fixed branch point, with corresponding ideal $\mathfrak{m}=\mathfrak{m}_{\nu}$ of $\mathcal{O}_{0}$. Fix also a prime $\mathfrak{M} \subset \mathcal{O}^{\prime}$ lying over $\mathfrak{m}$, with corresponding decomposition group $D=\{\sigma \in G$ : $\left.\mathfrak{M}^{\sigma}=\mathfrak{M}\right\}$, and inertia group $I=I(\mathfrak{M} / \mathfrak{m})$. Let $T$ be a subset of $G=G_{\lambda}=\operatorname{Gal}\left(K^{\prime} / K_{0}\right)$ such that

$$
G=\coprod_{\tau \in T} \mathcal{E} \tau D
$$

is the decomposition of $G$ into disjoint double cosets, where $\mathcal{E}=\operatorname{Gal}\left(K^{\prime} / E\right)$ is the subgroup fixing $E$.

As is clear from Lemma [3, it will be important to keep track of the primes $\mathfrak{n}$ of $\mathcal{O}_{E}$ dividing $\mathfrak{m}$ and especially their ramification indices $e(\mathfrak{n} / \mathfrak{m})$. That these can be described 
nicely in terms of the double coset decomposition (77) is a useful fact (we learned from Tate) for which we were not able to find a suitable reference, so we give the details. For each $\sigma \in G$, let $\mathfrak{n}_{\sigma}$ be the prime $\mathfrak{M}^{\sigma} \cap \mathcal{O}_{E}$ of $\mathcal{O}_{E}$ lying under $\mathfrak{M}^{\sigma}$. Let $I_{\sigma} \subseteq D_{\sigma}$ be the inertia and decomposition groups of $\mathfrak{M}^{\sigma} / \mathfrak{m}$, respectively. They satisfy $D_{\sigma}=\sigma D \sigma^{-1}$ and $I_{\sigma}=\sigma I \sigma^{-1}$. In the extension $K^{\prime} / E$, the inertia and decomposition groups for $\mathfrak{M}^{\sigma} / \mathfrak{n}_{\sigma}$ are simply $I_{\sigma} \cap \mathcal{E}$ and $D_{\sigma} \cap \mathcal{E}$, respectively. For the ramification indices of $\mathfrak{M} / \mathfrak{m}, \mathfrak{M} / \mathfrak{n}_{\sigma}$, and $\mathfrak{n}_{\sigma} / \mathfrak{m}$, let us put

$$
e=e(\mathfrak{M} / \mathfrak{m}), \quad e_{\sigma}^{\prime}=e\left(\mathfrak{M} / \mathfrak{n}_{\sigma}\right), \quad e_{\sigma}=e\left(\mathfrak{n}_{\sigma} / \mathfrak{m}\right),
$$

and similarly for the residual degrees, we put

$$
f=f(\mathfrak{M} / \mathfrak{m}), \quad f_{\sigma}^{\prime}=f\left(\mathfrak{M} / \mathfrak{n}_{\sigma}\right), \quad f_{\sigma}=f\left(\mathfrak{n}_{\sigma} / \mathfrak{m}\right) .
$$

By multiplicativity in towers for these invariants, we have

$$
e_{\sigma} e_{\sigma}^{\prime}=e, \quad f_{\sigma} f_{\sigma}^{\prime}=f .
$$

Lemma 4. With the notation introduced above,

(a) The distinct primes of $\mathcal{O}_{E}$ dividing $\mathfrak{m}$ are those induced by $\mathfrak{M}^{\tau}$ for $\tau \in T$. In other words, we have $\mathfrak{n}_{\sigma}=\mathfrak{n}_{\sigma^{\prime}}$ if and only if $\mathcal{E} \sigma D=\mathcal{E} \sigma^{\prime} D$.

(b) For $\sigma \in G$, we have

$$
e_{\sigma} f_{\sigma}=\left[\sigma D \sigma^{-1}: \mathcal{E} \cap \sigma D \sigma^{-1}\right], \quad e_{\sigma}=\left[\sigma I \sigma^{-1}: \mathcal{E} \cap \sigma I \sigma^{-1}\right] .
$$

Proof. Let $w$ be the valuation of $\mathcal{O}^{\prime}$ corresponding to $\mathfrak{M}$. For $\alpha \in \mathcal{O}^{\prime}$, we have $|\alpha|_{\sigma w}=$ $\left|\sigma^{-1} \alpha\right|_{w}$. If $\mathcal{E} \sigma D=\mathcal{E} \sigma^{\prime} D$, we can write $\sigma^{\prime}=h \sigma g$, with $h \in \mathcal{E}, g \in D$. For $\alpha \in \mathcal{O}_{E}$, we compute

$$
|\alpha|_{\sigma^{\prime} w}=|\alpha|_{h \sigma g w}=|\alpha|_{h \sigma w}=\left|h^{-1} \alpha\right|_{\sigma w}=|\alpha|_{\sigma w} .
$$

Thus, $\sigma w$ and $\sigma^{\prime} w$ induce the same valuation on $\mathcal{O}_{E}$, i.e. $\mathfrak{n}_{\sigma}=\mathfrak{n}_{\sigma^{\prime}}$. Conversely, suppose $\mathfrak{n}_{\sigma}=\mathfrak{n}_{\sigma^{\prime}}$, i.e. the set of primes of $\mathcal{O}^{\prime}$ lying over $\mathfrak{n}_{\sigma}$ includes $\mathfrak{M}^{\sigma^{\prime}}$ as well as $\mathfrak{M}^{\sigma}$. Since $\mathcal{E}=\operatorname{Gal}\left(K^{\prime} / E\right)$ acts transitively on this set, there exists $h \in \mathcal{E}$ such that $\mathfrak{M}^{h \sigma^{\prime}}=\mathfrak{M}^{\sigma}$, i.e.

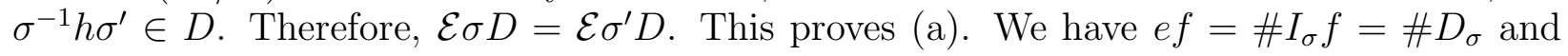
$e_{\sigma}^{\prime} f_{\sigma}^{\prime}=\#\left(I_{\sigma} \cap \mathcal{E}\right) f_{\sigma}^{\prime}=\#\left(D_{\sigma} \cap \mathcal{E}\right)$, so we get (b) by multiplicativity in towers (8) ).

Define

$$
Y=\left\{\sigma \in G: \sigma I \sigma^{-1} \subset \mathcal{E}\right\} .
$$

For the application to Riemann-Hurwitz, we'll need to estimate $c_{1}$. We proceed as follows.

Lemma 5. If $a \in Y$, then $\{b \in Y: \mathcal{E} a I=\mathcal{E} b I\}=\mathcal{E} a$. We have $c_{1}=\# Y / \# \mathcal{E}$.

Proof. We first make a remark that simplifies the calculation. Note that if we compose our fields $K_{0} \subset E \subset K^{\prime}$ with a finite extension $\tilde{K}$ of the constant field $K$ that splits $\mathfrak{M}$, then $c_{\delta}(\mathfrak{m})$ remains unchanged, since each prime $\mathfrak{n}_{\sigma}$ of $E$ of residual degree $f_{\sigma}$ splits in $E \tilde{K}$ into $f_{\sigma}$ primes of residual degree 1 with the same inertia group $I_{\sigma} \cap \mathcal{E}$. In fact, the genus calculation we are performing is a purely geometric one, so we could have simply assumed from the outset that the constant field $K$ is algebraically closed.

Either way, we take $\mathfrak{M} / \mathfrak{m}$ as above and assume without loss of generality, that $f(\mathfrak{M} / \mathfrak{m})=$ 1, i.e. $I=D$.

By Lemma 4, for any $\sigma \in G, e\left(\mathfrak{n}_{\sigma} / \mathfrak{m}\right)=1$ if and only if $\sigma I \sigma^{-1} \subset \mathcal{E}$. Thus

$$
c_{1}=\#\left\{\mathcal{E} \sigma I: \sigma I \sigma^{-1} \subset \mathcal{E}\right\} \text {. }
$$


Note that $\mathcal{E} a I=\mathcal{E} b I$ if and only if $b \in \mathcal{E} a I$. Suppose $b \in Y$ and $b \in \mathcal{E} a I$. Then $b a^{-1} \in \mathcal{E} a I a^{-1} \subset \mathcal{E}$, hence $b \in \mathcal{E} a$. Conversely, suppose $b=h a$ with $h \in \mathcal{E}$. Then

$$
b I b^{-1}=h a I a^{-1} h^{-1} \subset h \mathcal{E} h^{-1}=\mathcal{E}
$$

so $b \in Y$. Finally, clearly $\mathcal{E} a \subset \mathcal{E} a I$ so $b \in \mathcal{E} a$ implies $b \in \mathcal{E} a I$. Therefore, $Y$ is a union of (right) cosets of $\mathcal{E}$, and the number of distinct double cosets $\mathcal{E} a I$ with $a \in Y$ is exactly $\# Y / \# \mathcal{E}$. This completes the proof by (9).

Since we are working with function fields of characteristic 0, all ramification is tame, so the inertia group $I$ is cyclic. We now specialize to the case where $G=S_{n}$, and $I$ is generated by a cycle (under its natural action on the roots of $\lambda$ ). Of course, if \#I is greater than $n / 2$, the latter condition holds automatically.

Lemma 6. If $\operatorname{Gal}\left(K^{\prime} / K_{0}\right)=S_{n}$ and $I$ is generated by an m-cycle, then

$$
\begin{aligned}
c_{1} & =\frac{(\text { number of } m \text {-cycles in } \mathcal{E})}{\# \mathcal{E}} \times m(n-m) ! \\
& <m(n-m) !
\end{aligned}
$$

Proof. Just as in the proof of the preceding Lemma, we may assume that $I=D$. Let $J=\left\{s I s^{-1} \subset \mathcal{E}: s \in G\right\}$ be the set of subgroups of $\mathcal{E}$ which are $G$-conjugate to $I$. Then

$$
\# Y=\sum_{I^{\prime} \in J} \#\left\{s \in G: s I^{\prime} s^{-1}=I^{\prime}\right\} .
$$

Any two $m$-cycles in $S_{n}$ are $S_{n}$-conjugate, so

$$
\begin{aligned}
\# J & =\text { number of cyclic subgroups of } \mathcal{E} \text { generated by an } m \text {-cycle } \\
& =\text { (number of } m \text {-cycles in } \mathcal{E}) / \varphi(m) .
\end{aligned}
$$

There are $n ! /(m(n-m) !) m$-cycles in $S_{n}$, so for any $S_{n}$-conjugate $I^{\prime} \subset \mathcal{E}$ of $I$,

$$
\#\left\{s \in S_{n}: s I^{\prime} s^{-1}=I^{\prime}\right\}=\frac{n !}{\# \operatorname{orbit}_{S_{n}}\left(I^{\prime}\right)}=\frac{n !}{\frac{n !}{m(n-m) !} / \varphi(m)}=m \varphi(m)(n-m) ! .
$$

The proof is complete once we combine (12)-(15) with Lemma 5 ,

We end this section with an elementary criterion which guarantees the hypothesis of Lemma 6 (on inertia being generated by a cycle) to hold; the criterion will be easily verified for the Generalized Laguerre Polynomial at all its branch points.

Recall that $K_{1} / K_{0}$ is a root field for $\lambda$, i.e. $K_{1} \simeq K_{0}[x] /(\lambda)$.

Definition 2. Let $\nu \in B_{\lambda}$ be a branch point of $\lambda$, with corresponding maximal ideal $\mathfrak{m} \subset \mathcal{O}_{0}$. Let $e>1$ be an integer. We say that $\nu$ (or $\mathfrak{m}$ ) is simple of index $e$ for $\lambda$ if

$$
\mathfrak{m} \mathcal{O}_{K_{1}}=\mathfrak{n}_{0}^{e} \mathfrak{n}_{1} \cdots \mathfrak{n}_{s},
$$

where $\mathfrak{n}_{0}, \ldots, \mathfrak{n}_{s}$ are pairwise distinct primes of $\mathcal{O}_{K_{1}}$; in other words, in $\mathcal{O}_{K_{1}}$, there is a unique prime dividing $\mathfrak{m O}_{K_{1}}$ with non-trivial ramification index (equal to e).

Lemma 7. Suppose $G=\operatorname{Gal}\left(K^{\prime} / K_{0}\right)=S_{n}$. Let $\mathfrak{m} \subset \mathcal{O}_{0}$ be a maximal ideal corresponding to a branch point $\nu \in B_{\lambda}$, which is simple of index $e>1$. Then, for any $\mathfrak{M} \subset \mathcal{O}^{\prime}$ lying above $\mathfrak{m}$, the inertia group $I=I(\mathfrak{M} / \mathfrak{m})$ has order $e$ and is generated by a cycle of length $e$. 
Proof. Let $\mathcal{E}=\operatorname{Gal}\left(K^{\prime} / K_{1}\right)$. The index $n$ subgroups in $S_{n}$ are stabilizers of any one of the $n$ letters. By reordering the roots if needed, we can identify $\mathcal{E} \simeq S_{n-1}$ with the stabilizer of the letter $n$. Every element in $S_{n}$ is a product of disjoint, non-trivial cycles. This decomposition is unique once a labelling is fixed, and two elements in $S_{n}$ are conjugate if and only if they decompose into the same number of cycles of each length.

Returning to the proof of the Lemma, suppose $\mathfrak{M}$ is a prime of $\mathcal{O}^{\prime}$ whose restriction $\mathfrak{M} \cap \mathcal{O}_{K_{1}}$ is the unique prime $\mathfrak{n}$ of $\mathcal{O}_{K_{1}}$ of ramification index $e>1$ over $\mathfrak{m}$. Let $I=I(\mathfrak{M} / \mathfrak{m})$. We may assume, as in the preceding lemmas, that composing with a suitable finite extension of $K, \mathfrak{M} / \mathfrak{m}$ has degree 1, i.e. $I=D$ (this disturbs neither the identification $G \simeq S_{n}$ nor the embedding $I \hookrightarrow G$ ).

Let $\gamma$ be a generator of the cyclic group $I$. Write $\gamma=\gamma_{1} \cdots \gamma_{r}$ for its decomposition into disjoint, possibly trivial, cycles. Since the $\gamma_{i}$ pairwise commute, we may assume that the letter $n$ occurs in the cycle $\gamma_{1}$. For $1 \leq i \leq r$, let $a_{i}=\operatorname{ord}\left(\gamma_{i}\right) \geq 1$, and let $a=$ $\min \left\{m \geq 1: \gamma^{m} \in \mathcal{E}\right\}$. On the one hand, $\gamma^{a}$ generates $I \cap \mathcal{E}$, and, on the other hand, we have $a=a_{1}$ (recalling our convention that $\mathcal{E}$ is the stabilizer of the letter $n$ ). By Lemma 4 , $e(\mathfrak{n} / \mathfrak{m})=\#[I /(I \cap \mathcal{E})]=\#\left[\langle\gamma\rangle /\left\langle\gamma^{a}\right\rangle\right]=a$, thus $\gamma_{1}$ has order $a=a_{1}=e>1$, since we took $\mathfrak{n}$ to be the unique prime of ramification index $e>1$ over $\mathfrak{m}$.

It remains to show that the cycles $\gamma_{2}, \ldots, \gamma_{r}$ are trivial, i.e. $a_{i}=1$ for $i>1$. We proceed by contradiction. If $a_{2}>1$, say, then, there exists $\sigma \in G$ such that $\sigma \gamma_{2} \sigma^{-1}$ is a cycle acting non-trivially on the letter $n$. Then, as before, $e\left(\mathfrak{n}_{\sigma} / \mathfrak{m}\right)=a_{2}>1$, so we get $a_{2}=e$ and $\mathfrak{n}_{\sigma}=\mathfrak{n}$ by the assumption on the simplicity of the ramification. By Lemma 4 , therefore, $\sigma \in \mathcal{E} I$, say $\sigma=\eta \theta$ with $\eta \in \mathcal{E}$ and $\theta \in I$. Letting $x^{\prime}=\sigma x \sigma^{-1}$ for $x \in G$, we have $\gamma^{\prime}=\gamma_{1}^{\prime} \gamma_{2}^{\prime} \cdots \gamma_{r}^{\prime}$ is the decomposition of $\gamma^{\prime}$ into disjoint cycles since conjugation preserves cycle structure. But we claim that $\gamma_{1}^{\prime}$ and $\gamma_{2}^{\prime}$ are not disjoint, as they both act non-trivially on the letter $n$. To see this, note that $\theta=\gamma^{b}$ for some integer $b$, so $\theta \gamma_{i} \theta^{-1}=\gamma_{i}$ for $i=1, \ldots, r$. On the other hand, since $\eta \in \mathcal{E}$, it fixes $n$, so $\gamma_{1}^{\prime}=\eta \gamma_{1} \eta^{-1}$ and $\gamma_{2}^{\prime}=\eta \gamma_{2} \eta^{-1}$ are both $e$-cycles that act non-trivially on $n$, hence are not disjoint. This contradiction shows that $\gamma_{2}, \ldots, \gamma_{r}$ are all trivial, so $I=\left\langle\gamma_{1}\right\rangle$ is generated by an $e$-cycle, hence has order $e$.

\section{Specializations of $S_{n}$-COVERS}

In this section, we develop a strategy for applying Proposition 1 to a geometric $S_{n}$-cover. Namely, starting with an $S_{n}$-extension of function fields $K^{\prime} / K_{0}$ as in section 2, in subsection 5.1] we construct a family of polynomials $\Lambda_{j}(x, t) \in K[x, t]$ with splitting field contained in $K^{\prime}$ (to which we will later apply Proposition 1). In 5.2, we will give a geometric interpretation in terms of fiber products for the curves corresponding to these $\Lambda_{j}$ which we need for controlling the genus of subfields of $K^{\prime}$ cut out by a subgroup contained in $A_{n}$. A reader who is interested in a proof of Theorem 1 for $n \geq 10$ only, can skip 5.2 entirely, as it will enter the proof only for $6 \leq n \leq 9$.

\subsection{Distinguished subfields in $S_{n}$-extensions.}

Let $\lambda(x, t)$ and $K^{\prime} / K_{0}$ be as in section 2 , in particular, recall the regularity hypothesis (i) introduced there. Suppose further that

(ii) $G_{\lambda} \simeq S_{n}$, and 
(iii) $\lambda$, as a polynomial in $x$, has discriminant which is non-constant in $t$.

These two conditions actually recover the regularity of the cover, at least when $n$ is not too small.

Lemma 8. Suppose $n \geq 5$. Then

(a) $K$ is algebraically closed in $K^{\prime} / K_{0}$, and

(b) $K^{\prime} / K_{0}$ has a unique Galois subfield. This subfield is quadratic over $K_{0}$.

Proof. Fix an algebraic closure $\bar{K}$ of $K$. Then $\bar{K} \cap K^{\prime}$ is a Galois subfield of the $S_{n^{-}}$extension $K^{\prime} / K_{0}$. Since $n \geq 5$, the only non-trivial Galois subfield in $K^{\prime} / K_{0}$ is the unique quadratic subfield generated by the square-root of the discriminant (with respect to $x$ ) of $\lambda(x, t)$. Invoke the discriminant condition on $\lambda$ and we are done.

The following result is standard.

Lemma 9. Let $X / K$ be a smooth projective curve, and let $\xi: X \rightarrow \mathbf{P}_{K}^{1}$ be a non-constant $K$-morphism. Then $X$ is $K$-birational to a plane curve $G(x, t)=0$ such that $\xi$ is the projection-to-t map.

We now describe a distinguished collection of subfields in $K^{\prime} / K_{0}$. Fix a labelling of the roots of $\lambda(x, t)$ over $K_{0}$, giving an identification of $G_{\lambda}$ with the symmetric group $S_{n}$. For $1 \leq j<n$, write $S_{n, j}$ for the subgroup $S_{j} \times S_{n-j} \subset S_{n}$, where $S_{j}$ permutes the first $j$ roots, and $S_{n-j}$, the remaining $n-j$ roots. Denote by

- $K_{j}$ the subfield of $K^{\prime} / K_{0}$ fixed by $S_{n, j}$,

- $X_{j}$ the associated smooth projective curve over $K$, and

- $\tilde{\phi}_{n, j}: X_{j} \rightarrow \mathbf{P}_{K}^{1}$ the $K$-branched cover corresponding to the extension $K_{j} / K_{0}$.

Lemma 9 furnishes a $K$-birational map taking $X_{j}$ to a plane curve $\Lambda_{j}(x, t)=0$ which is smooth above $t=\beta_{0}$, and such that $\tilde{\phi}_{n, j}$ is the projection-to- $t$ map. Clearly we can take $\Lambda_{1}=\lambda$ and do so. Since $X_{j}$ is smooth, it is absolutely irreducible, hence so is $\Lambda_{j}(x, t)$. Thus we can apply Proposition 1 to $\Lambda_{j}$.

Lemma 10. Fix positive integers $n, j$ satisfying $n \geq 5$ and $j \in[1, n / 2]$. Suppose for every intermediate subfield $E$ of $K^{\prime} / K_{0}$ over which $\Lambda_{j}(x, t)$ is reducible, we have $g\left(X_{E}\right)>1$. Then for $\alpha \in \in_{a f} K$, the specialization $\lambda(x, \alpha)$ is $K$-irreducible, and its splitting field has degree divisible by $\left(\begin{array}{l}n \\ j\end{array}\right)$.

Proof. As $\operatorname{deg} \tilde{\phi}_{n, j}=\left[K_{j}: K_{0}\right]=\# S_{n} / \# S_{n, j}=\left(\begin{array}{c}n \\ j\end{array}\right) \geq n$, and $n \geq 5$, Lemma 8 (b) says that $K^{\prime} / K_{0}$ is the Galois closure of $K_{j}$; equivalently, $K^{\prime} / K_{0}$ is the splitting field of $\Lambda_{j}(x, t)$ over $K_{0}$. But $K^{\prime}$ is the splitting field of $\lambda(x, t)=\Lambda_{1}(x, t)$ over $K_{0}$, so by Proposition [1, for $\alpha \in_{\mathrm{af}} K$ the splitting field of $\lambda(x, \alpha)$ contains the roots of $\Lambda_{j}(x, \alpha)$, and we are done.

For the proof of Theorem 11 we will employ the following application of Proposition 1

Theorem 3. Suppose $n \geq 7$ and $\Lambda_{j}(x, t)$ satisfies the hypothesis in Lemma 10 for each integer $j \in[1, n / 2]$. Then for $\alpha \in_{a f} K$, the specialization $\lambda(x, \alpha)$ is $K$-irreducible and has Galois group containing $A_{n}$. 
Proof. First, recall that $\Lambda_{1}=\lambda$. By Lemma 10, $\lambda(x, \alpha)$ is $K$-irreducible for $\alpha \in \in_{\text {af }} K$, hence its Galois group is a transitive subgroup of $S_{n}$. If $n \geq 8$, then there exists a prime $q$ with $n / 2<q<n-2$ [25, p. 370]. Necessarily $q$ divides $\left(\begin{array}{l}n \\ k\end{array}\right)$ for some $1<k<n / 2$, so by Lemma 10. for $\alpha \in_{\mathrm{af}} K$ the specialization $\lambda(x, \alpha)$ is $K$-irreducible, and $q$ divides the degree of its splitting field over $K$. That means the Galois group of such a $\lambda(x, \alpha)$ is a transitive subgroup of $S_{n}$ and has order divisible by $q$; a theorem of Jordan [17, Thm 5.6.2 and 5.7.2] then implies that this Galois group contains $A_{n}$.

For $n=7$, Lemma 10 implies that for $\alpha \in$ af $K$, the Galois group of $\lambda(x, \alpha)$ is a transitive subgroup of $S_{7}$ of size divisible by $\operatorname{LCM}\left(\left(\begin{array}{l}7 \\ 2\end{array}\right),\left(\begin{array}{l}7 \\ 3\end{array}\right)\right)=105$. By the classification of transitive subgroups of $S_{7}$ [7, p. 60] it follows that this Galois groups contains $A_{7}$.

\subsection{Interpretation in terms of fiber products.}

We continue with the notation of the previous subsection and assume properties (i)-(iii) are satisfied. Fix a labelling $\lambda_{1}, \ldots, \lambda_{n}$ of the roots of $\lambda=\Lambda_{1}$ in $K^{\prime}$, and let $\Sigma=\Sigma_{1}=$ $\left\{\lambda_{1}, \ldots, \lambda_{n}\right\}$. For an integer $j \in[1, n-1]$, let $\Sigma_{j}$ be the set of roots of $\Lambda_{j}$ in $K^{\prime}$, and let $\Sigma^{(j)}$ be the set of " $j$-subsets" of $\Sigma$ (i.e. those of cardinality $j$ ). Recall that $\Lambda_{j}$ splits into linear factors over $K^{\prime}$, hence $\# \Sigma_{j}=\# \Sigma^{(j)}=\left(\begin{array}{c}n \\ j\end{array}\right)$. Each of these sets carries a natural action of $\operatorname{Gal}\left(K^{\prime} / K_{0}\right) \simeq S_{n}$

Lemma 11. For each $j \in[1, n-1]$, there is a bijective correspondence between $\Sigma_{j}$ and $\Sigma^{(j)}$ which respects the natural action of $\mathrm{Gal}\left(K^{\prime} / K_{0}\right)$ on these sets.

Before proving Lemma 11, let us state two applications of it that we shall need.

Proposition 2. For $\alpha \in K$, the $K$-rational roots of $\Lambda_{j}(x, \alpha)$ are in one-to-one correspondence with the $K$-rational degree $j$ factors of $\lambda(x, \alpha)$.

Proof. The $K$-rational linear factors of $\Lambda_{j}(x, \alpha)$ are in one-to-one correspondence with the fixed points of $G_{\lambda}$ in its action on $\Sigma^{(j)}$. By the Lemma, these are in one- to-one correspondence with the $G_{\lambda}$-invariant subsets of $\Sigma$ of size $j$. The roots of a $K$-rational degree $j$ factor of $\lambda(x, \alpha)$ clearly form such a subset, and conversely, a $G_{\lambda}$-invariant $T \in \Sigma_{j}$ gives the $K$-rational degree $j$ factor $\prod_{\theta \in T}(x-\theta)$ of $\lambda$.

Remark 5. Proposition 2 lends some perspective on Proposition [1. Namely, $\lambda$ has a degree $j$ factor, $1 \leq j \leq n-1$, over some intermediate field $K_{0} \subseteq E \subseteq K^{\prime}$ if and only if $\Lambda_{j}$ has a root in $E$, i.e. if and only if $E$ contains (a conjugate of) $K_{j}$. Thus the hypothesis of Proposition 1 , namely that $g\left(X_{E}\right) \geq 2$ for every $E$ over which $\lambda$ is reducible is equivalent to the hypothesis that $g\left(X_{j}\right) \geq 2$ for $1 \leq j \leq n-1$. One then obtains Proposition 1 by applying Proposition 2 in conjunction with Faltings' Theorem.

Proposition 3. Suppose $1 \leq j \leq n-1$. Then $\Lambda_{j}(x, t)$ is irreducible over the subfield of $K^{\prime} / K_{0}$ fixed by $A_{n}$.

Proof of Proposition 3 . Since $A_{n}$ is $(n-2)$-transitive, if $2 \leq j \leq n-2$ then $A_{n}$, as a subgroup of the group of permutations on the set $\Sigma$, acts transitively on the set $\Sigma^{(j)}$. Thanks to Lemma 11. $A_{n}$, as a subgroup of $\operatorname{Gal}\left(K^{\prime} / K_{0}\right)$, then acts transitively on the set of roots of $\Lambda_{j}$ in $K^{\prime}$, establishing the Proposition for this range of $j$. 
Write $F$ for the fixed field of $K^{\prime} / K_{0}$ by $A_{n}$. If $\Lambda_{1}$ is reducible over $F$, then $\operatorname{Gal}\left(K^{\prime} / F\right)$ is contained in $S_{l} \times S_{n-l}$ for some $1 \leq l \leq n-1$. Since $F / K_{0}$ is quadratic, \#Gal $\left(K^{\prime} / K_{0}\right) \leq$ $2 \cdot \# S_{l} \cdot \# S_{n-l}<\# S_{n}$, a contradiction. Thus $\Lambda_{1}$ is irreducible over $F$. Thanks to Lemma 11. that means $A_{n}$, as a subgroup of the group of permutations of $\Sigma$, acts transitively on $\Sigma^{(1)}$, hence also on $\Sigma^{(n-1)}$. Applying Lemma 11] again, we see that $\Lambda_{n-1}$ is irreducible over $F$, as desired.

We now verify Lemma 11] via a fiber product construction. The Lemma and the construction are probably well-known, but we cannot locate a reference for either one so we give the details here. We begin with a general setup. Recall that $K$ is a field of characteristic 0 .

Let $\wp_{K}^{n}$ denote the set of equivalence classes of non-zero, degree $\leq n$ polynomials in $K[x]$, where two polynomials are identified if they are $K^{\times}$-multiples of each other. We have a natural bijection between $\wp_{K}^{n}$ and the set of $K$-rational points $\mathbf{P}_{K}^{n}(K)$ of projective $n$-space, via

$$
a_{0} x^{n}+a_{1} x^{n-1}+\cdots+a_{n} \longmapsto\left[a_{0}: \cdots: a_{n}\right] .
$$

In light of this, to give a polynomial $\lambda(x, t) \in K[x, t]$ which is non-constant and of degree $\leq n$ in $x$ is to give a non-constant $K$-morphism $\Lambda: \mathbf{P}_{K}^{1} \rightarrow \mathbf{P}_{K}^{n}$. Also, for every $1 \leq j<n$ the multiplication map $\wp_{K}^{j} \times \wp_{K}^{n-j} \rightarrow \wp_{K}^{n}$ gives rise to a $K$-morphism $\phi_{n, j}: \mathbf{P}_{K}^{j} \times \mathbf{P}_{K}^{n-j} \rightarrow \mathbf{P}_{K}^{n}$, whence a pull-back diagram

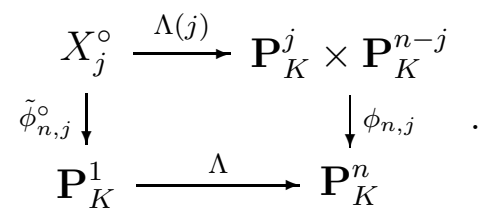

Denote by $\phi_{n}:\left(\mathbf{P}_{K}^{1}\right)^{n} \rightarrow \mathbf{P}_{K}^{n}$ the $K$-morphism corresponding to the $n$-fold multiplication map $\left(\wp_{K}^{1}\right)^{n} \rightarrow \wp_{K}^{n}$. Then we have an analogous pull-back diagram

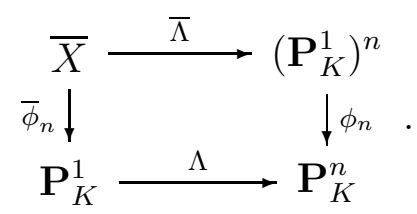

Any permutation of the $n$-coordinates of the points of $\left(\mathbf{P}_{K}^{1}\right)^{n}$ is a $K$-morphism which is compatible with $\phi_{n}$. Clearly $\operatorname{deg} \phi_{n}=n$ !, so $\phi_{n}$ is a regular branched cover with deck transformation group $S_{n}$.

Suppose $\Lambda$ corresponds to a separable, degree $n$ polynomial $\lambda$ over $K_{0}=K(t)$. Then the fiber of $\bar{\phi}_{n}$ over the generic point of $\mathbf{P}_{K}^{1}$ consists of $n$ ! pairwise distinct, ordered $n$-tuples of the roots of $\lambda$ over $K_{0}$. Every element of the Galois group $G_{\lambda}$ of $\lambda$ over $K_{0}$ permutes these $n$-tuples, and such a permutation gives rise to a permutation on $\left(\mathbf{P}_{K}^{1}\right)^{n}$ making the diagram (18) commute. Having fixed a labelling of the roots of $\lambda$, we see that $G_{\lambda}$ is canonically identified with a subgroup of $S_{n}$. Since $\lambda$ is separable over $K_{0}$, these $n ! n$-tuples are pairwise 
distinct, whence the scheme $\bar{X}$ is reduced. Also,

$$
\begin{aligned}
\bar{X} \text { is } K \text {-reducible } & \Leftrightarrow \text { the generic fiber of } \bar{\phi}_{n} \text { is the disjoint union } \\
& \Leftrightarrow \text { of non-trivial, } G_{\lambda} \text {-stable subsets } \\
& \Leftrightarrow G_{\lambda} \text { does not act transitively on this fiber } \\
& \Leftrightarrow G_{\lambda} \subsetneq S_{n} .
\end{aligned}
$$

The multiplication map $\left(\wp_{K}^{1}\right)^{n} \rightarrow \wp_{K}^{n}$ naturally factors through every $\wp_{K}^{j} \times \wp_{K}^{n-j}$. That means $\bar{\phi}_{n}$ factors through $\phi_{n, j}$ for every $j$; therefore the diagram (18) factors through the diagram (17) for every $j$, and $\Phi_{n, j}$ is also a regular branched cover with deck transformation group isomorphic to $S_{j} \times S_{n-j}$ :

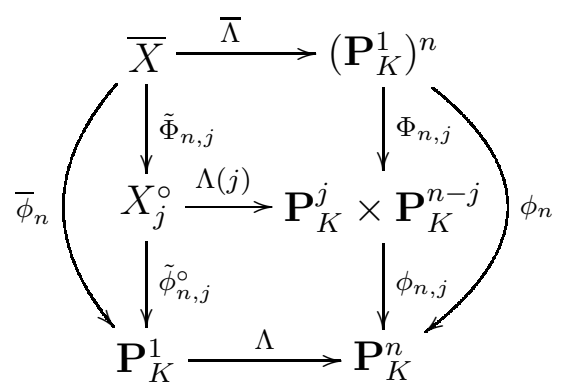

Finally, suppose $\lambda$ satisfies hypotheses (i)-(iii). Then $G_{\lambda}=S_{n}$, whence the deck transformation group of $\bar{\phi}_{n}$ is also $S_{n}$. By (19), the scheme $\bar{X}$ is reduced and $K$-irreducible, and so it makes sense to speak of the function field $K(\bar{X})$. Both $\tilde{\Phi}_{n, j}$ and $\tilde{\phi}_{n, j}$ are surjective, so $X_{j}^{\circ}$ is also $K$-irreducible, and so it makes sense to speak of the function field $K\left(X_{j}^{\circ}\right)$ as well, and $K(\bar{X}) / K\left(\mathbf{P}_{K}^{1}\right)$ is an $S_{n}$-extension of function fields. We have $\operatorname{deg} \tilde{\Phi}_{n, j}=j !(n-j)$ !, and the same argument after (18) shows that the deck transformation group of $\tilde{\Phi}_{n, j}$ is isomorphic to a subgroup of, and hence is exactly, $S_{j} \times S_{n-j}$.

Proof of Lemma 11. First, recall the notations $X_{j}, K_{j}$ etc. introduced after Lemma 9] and the fact that $X_{1}$ is given by $\lambda=0$. For any $t_{0} \in \mathbf{P}_{\bar{K}}^{1}$, the $\bar{K}$-rational points on the fibers of $\tilde{\phi}_{n, 1}^{\circ}$ are in bijective correspondence with the $\bar{K}$-linear factors of $\lambda\left(x, t_{0}\right)$, while those on the fibers of $\tilde{\phi}_{n, 1}$ are in bijective correspondence with the $\bar{K}$-rational points of the curve $\lambda(x, t)=0$ with $t$-coordinates $t_{0}$. These two sets are in natural bijective correspondence with each other; the universal property of the pullback diagram (17) then implies that there is a $K$-isomorphism $\mu_{n}: X_{1} \rightarrow X_{1}^{\circ}$ such that $\tilde{\phi}_{n, 1}=\mu_{n} \tilde{\phi}_{n, 1}^{\circ}$. This allows us to identify the two $S_{n}$-extensions $K(\bar{X}) / K_{0}$ and $K^{\prime} / K_{0}$. The $S_{j} \times S_{n-j}$ subgroups in $S_{n}$ are pairwise conjugate, so we can identify the intermediate subfields $K\left(X_{j}^{\circ}\right)$ with $K\left(X_{j}\right)$. That means the smooth curve $X_{j}$ is the canonical desingularization of $X_{j}^{\circ}$, and $\tilde{\phi}_{n, j}$ is the extension of $\tilde{\phi}_{n, j}^{\circ}$ to $X_{j}$, whence the $\operatorname{Gal}\left(K^{\prime} / K_{0}\right) \simeq S_{n}$ action on the roots of $\Lambda_{j}$ over $K_{0}$ is the same as that on the generic fiber of $\tilde{\phi}_{n, j}^{\circ}$. But the points on this generic fiber are precisely the $j$-subsets of $\Sigma$.

\section{Generalized Laguerre Polynomials}

In this section we apply the machinery developed above to study specializations of Generalized Laguerre Polynomials $L_{n}^{(t)}(x)$ defined in the introduction. In subsection 6.1, we study the singular locus of the plane curve $\mathcal{L}_{n}$ defined by $L_{n}^{(t)}(x)=0$. By analyzing the structure 
of maximal subgroups of $S_{n}$, in subsection 6.2 we compute the genus of the intermediate subfields of $K^{\prime} / K_{0}$ over which $\Lambda_{j}$ is reducible. In subsection 6.3 we combine these ingredients to deduce Theorem 1 following the strategy outlined in sections 2 and 5 .

From now on, we fix $n$ and take $\lambda(x, t)=L_{n}^{(t)}(x)$, carrying over all the notation $\left(K_{0}, K_{1}\right.$, $K^{\prime}, \mathcal{O}_{0}, G_{\lambda}, B_{\lambda}$, etc.) from sections 2, 4, 5 to the present setting.

\subsection{The singular locus of $L_{n}^{(t)}(x)$.}

Fix $n>2$. Following Schur [27, p. 54], we homogenize $L_{n}^{(t)}(x)$ by setting

$$
\begin{aligned}
F_{n}(x, \nu, \mu) & :=(-1)^{n} n ! \mu^{n} L_{n}^{(\nu / \mu)}(x / \mu) \\
& =x^{n}-\frac{k_{n}}{1} x^{n-1}+\frac{k_{n-1} k_{n}}{1 \cdots 2} x^{n-2}-\cdots+(-1)^{n} \frac{k_{1} \cdots k_{n}}{1 \cdot 2 \cdots n},
\end{aligned}
$$

where $k_{j}=j(\nu+j \mu)$. Let $\mathcal{L}_{n}$ be the plane curve $F_{n}(x, \nu, \mu)=0$. To simplify the notation, we write $\partial_{x} F_{j}$ for $\partial F_{j} / \partial x$. Then we have the relations [27, p. 54]

$$
\begin{aligned}
x \partial_{x} F_{m} & =m F_{m}+k_{m} F_{m-1}, & & \left(m \geq 1, F_{0}:=1\right) ; \\
F_{m} & =(x-\nu-(2 m-1) \mu) F_{m-1}-\mu k_{m-1} F_{m-2}, & & (m \geq 2) .
\end{aligned}
$$

Setting $\mu=0$, (201) becomes

$$
x^{n}-n x^{n-1} \nu+\frac{n(n-1)}{2} x^{n-2} \nu^{2}-\cdots+(-1)^{n} \nu^{n}=(x-\nu)^{n} .
$$

Thus $\mathcal{L}_{n}$ has exactly one point along the line at infinity, namely [1:1:0]. Let $\iota_{n}: \mathcal{L}_{n} \rightarrow \mathbf{P}_{K}^{1}$ be the projection map defined by $[x: \nu: \mu] \mapsto[\nu: \mu]$.

Lemma 12. Suppose for some integer $j \in[0, n]$ and some point $z=[x(z): \nu(z): \mu(z)] \in \mathbf{P}_{\mathbf{C}}^{2}$ with $x(z) \mu(z) \neq 0$, we have

$$
\left.F_{n-j}\right|_{z}=\left.\partial_{x} F_{n-j}\right|_{z}=0 \text { and } k_{n-j} \neq 0 .
$$

Then $\left.F_{n-j-1}\right|_{z}=0$ and $k_{n-j-1} \neq 0$. Moreover, if $j \leq n-2$, then $\left.\partial_{x} F_{n-j-1}\right|_{z}=0$.

Proof. Since $\mu(z) \neq 0$, without loss of generality we can set $\mu(z)=1$.

Suppose $n \geq j+1$; then substitute into (21) the first two relations in (23), we get $0=\left.k_{n-j} F_{n-j-1}\right|_{z}$, whence

$$
\left.F_{n-j-1}\right|_{z}=0
$$

Next, suppose $k_{n-j-1}=0$. When we use the expansion (20) to evaluate (24), we see that $x(z)=0$, a contradiction. Finally, suppose $n \geq j+2$. Substituting (24) along with the first relation in (23) into (22), we get

$$
0=-\left.\mu(z) k_{n-j-1} F_{n-j-2}\right|_{z} .
$$

Substitute this and (24) back into (21) and we get $\left.x \partial_{x} F_{n-j-1}\right|_{z}=0$. As $x(z) \neq 0$, that means $\left.\partial_{x} F_{n-j-1}\right|_{z}=0$. This completes the proof of the Lemma.

Lemma 13. For $n \geq 3$ the curve $\mathcal{L}_{n}$ has no finite singular point. 
Proof. Using the relations (21) and (22), Schur [27, p. 54] showed that $F_{n}$, viewed as a polynomial in $x$, has discriminant

$$
\mu^{\frac{n(n-1)}{2}} n ! k_{2} k_{3}^{2} \cdots k_{n}^{n-1} .
$$

We are interested in the finite points on $\mathcal{L}_{n}$, so for the rest of the proof we can set $\mu=1$. Clearly it suffices to consider only the points on $\mathcal{L}_{n}$ lying above the branch locus of $\iota_{n}$.

Suppose $z=\left(x_{0}, \nu_{0}\right)$ is a finite singular point. By (25) we have $\nu_{0} \in\{-2, \ldots,-n\}$, and

$$
\left.F_{n}\right|_{z}=\left.\partial_{x} F_{n}\right|_{z}=\left.\partial_{\nu} F_{n}\right|_{z}=0
$$

We claim that $x_{0} \neq 0$. Suppose otherwise; set $\partial_{\nu} F_{n}=0$ and then substitute $x=0$ (recall that $\mu=1$ ), to get

$$
0=(-1)^{n} \frac{\partial}{\partial \nu} \prod_{k=1}^{n}(\nu+k)=(-1)^{n} \sum_{\substack{m=1 \\ n}}^{n}(\nu+k) .
$$

Set $\nu=\nu_{0}$ and this becomes

$$
\prod_{\substack{k=1 \\ k \neq-\nu_{0}}}^{n}\left(\nu_{0}+k\right)=0
$$

a contradiction. Thus $x_{0} \neq 0$. Also, if $k_{n}=0$, then from (20) we get $x_{0}=0$, a contradiction. Thus $k_{n} \neq 0$, i.e. $\nu_{0} \neq-n$. That means the hypotheses of Lemma 12 are satisfied for $j=0$. Applying the lemma, we find the conditions of the lemma hold for $j=1$ as well as $\nu_{0} \neq 1-n$. Repeating this procedure, we find $\nu_{0} \notin\{-2, \cdots,-n\}$, a contradiction. Thus $\mathcal{L}_{n}$ has no finite singular point.

Lemma 14. Suppose $n \geq 2$. Then $K\left(\sqrt{\operatorname{disc} L_{n}^{(t)}(x)}\right)$ is a quadratic extension of $K_{0}$ corresponding to a smooth curve of genus $\left[\frac{n-2}{4}\right]$.

Proof. Since $n \geq 2$, (25) says that disc $L_{n}^{(t)}(x)$ is a polynomial in $t$ whose square-free part has degree $\left[\frac{n}{2}\right]$, and the Lemma follows.

Recall that the notation of section 4 , such as $\mathcal{O}_{0}, \mathcal{O}^{\prime}$ etc. now applies to the case $\lambda(x, t)=$ $L_{n}^{(t)}(x)$. For $\nu \in B_{\lambda}=\{-2, \ldots,-n\} \subset \mathbf{P}_{K}^{1}$, denote by $\mathfrak{m}_{\nu}$ the corresponding maximal ideal in $\mathcal{O}_{0}$. Denote by $\mathcal{O}_{1}$ the coordinate ring of an affine open set of $X_{1}$ containing all places lying above every $\nu$ with respect to the projection map $\iota_{n}$. Then (25) says that the restriction of $\iota_{n}$ to $\mathcal{O}_{0}$ is unramified outside the $\mathfrak{m}_{\nu}$, and Lemma 13] says that the inclusion map $\mathcal{O}_{0} \subset \mathcal{O}^{\prime}$ is an integral extension of Dedekind domains when localized at these $\mathfrak{m}_{\nu}$. From (20) and (25), we see that $\mathcal{O}_{1}$ has exactly one ramified maximal ideal lying above $\mathfrak{m}_{\nu}$ :

$$
\mathfrak{m}_{\nu} \mathcal{O}_{1}=\mathfrak{n}_{0}^{|\nu|} \mathfrak{n}_{1} \cdots \mathfrak{n}_{s}
$$

where the $\mathfrak{n}_{i}$ are pairwise distinct; in other words each branch point $\nu$ of $L_{n}^{(t)}(x)$ is simple of index $|\nu|$. Applying Lemma [7, we deduce the following result. 
Lemma 15. For $\nu \in\{-2, \ldots,-n\}$, let $\mathfrak{M}_{\nu} \subset \mathcal{O}^{\prime}$ be a maximal ideal lying above $\mathfrak{m}_{\nu}$. Then the inertia group $I\left(\mathfrak{M}_{\nu} / \mathfrak{m}_{\nu}\right)$ is generated by a cycle of length $|\nu|$. In particular,

$$
e_{\nu}:=e\left(\mathfrak{M}_{\nu} / \mathfrak{m}_{\nu}\right)=|\nu|
$$

Proposition 4. Suppose $n \geq 6$. Then the geometric genus of $\mathcal{L}_{n}$ is $>1$.

Proof. First, assume $n \geq 7$. Thanks to Lemma 15, we can apply Lemma 3 with $V=$ $\{-n, 1-n, \ldots, 5-n\}$. Since there is a unique prime in $\mathcal{O}_{1}$ above $\mathfrak{m}_{\nu}$ with non-trivial ramification index $-n u$, we have $c_{1}(\nu)=n+\nu$, and (4) becomes

$$
g\left(\mathcal{L}_{n}\right)=g\left(K_{1}\right) \geq 1+\frac{n}{2}\left(-2+\sum_{i=0}^{5}\left(1-\frac{1}{d(n-i)}\right)\right)-\frac{1}{2} \sum_{i=0}^{5}\left(1-\frac{1}{d(n-i)}\right) \cdot i
$$

For any six consecutive, positive integers, exactly two of them are prime to 6 , another one is odd, and the remaining three are even. Thus the first $i$-sum in (28) is

$$
\geq-2+6-2 \times \frac{1}{5}-\frac{1}{3}-3 \times \frac{1}{2}=\frac{53}{30} .
$$

Thus (28) yields

$$
g\left(\mathcal{L}_{n}\right) \geq 1+\frac{53 n}{60}-\frac{1}{2}\left(1-\frac{1}{n}\right)(0+1+2+3+4+5),
$$

which is $>1$ if $n>10$. Using the more refined version (28) we find that in fact $g\left(\mathcal{L}_{n}\right)>1$ if $n \geq 7$. Using the full Riemann-Hurwitz formula, or the AlgCURVES package in MAPLE, we find that $g\left(\mathcal{L}_{6}\right)=4$. This completes the proof of the Proposition.

Remark 6 . By analyzing the singularity at infinity, one in fact has a nice formula $g\left(\mathcal{L}_{n}\right)=$ $\left[(n-2)^{2} / 4\right]$ valid for all $n$.

\subsection{Genus of maximal subgroups.}

In this subsection, we carry out the calculations which will be necessary ingredients for the application of Theorem 3 to $L_{n}^{(t)}(x)$ in the next section. This involves a mixed strategy in the following sense. For $n \geq 10$, we show that every minimal intermediate subfield $E$ of $K^{\prime} / K_{0}$ has genus $>1$, thanks to Lemma 14 and Proposition 5 below. It then follows from Riemann-Hurwitz that every proper intermediate subfield has genus $>1$. For $6 \leq n \leq 9$, the quadratic extension inside $K^{\prime} / K_{0}$ has genus 0 or 1 , but we have shown in Proposition 3 that $\Lambda_{j}$ is not reducible over this field. It remains, then, to check for $6 \leq n \leq 9$ that proper subgroups of $A_{n}$ give fixed fields of genus $>1$, and this is the content of Proposition [6. We treat $n=5$ "by hand."

Proposition 5. Suppose $n \geq 6$. If $\mathcal{E}$ is a maximal subgroup of $G_{\lambda}$ other than $A_{n}$, with corresponding fixed field $E$, then $g\left(X_{E}\right)>1$.

Proposition 6. Suppose $6 \leq n \leq 9$. If $\mathcal{E}$ is a proper maximal subgroup of $A_{n} \subset G_{\lambda}$, with corresponding fixed field $E$, then $g\left(X_{E}\right)>1$. 
Proof of Proposition 5. Up to conjugation, the maximal subgroups of $S_{n}$ other than $A_{n}$ belong to exactly one of the following three types [7, p. 268]:

- imprimitive subgroups: the wreath products $S_{j} 2 S_{n / j}$ in its imprimitive action ${ }^{1}$, for some divisor $j$ of $n, 1<j<n$;

- intransitive subgroups: $S_{n, j}$ for some $1 \leq j<n / 2$ (note that if $n$ is even then $S_{n, n / 2}$ is contained in $S_{n / 2}\left(S_{2}\right)$;

- a primitive subgroup of $S_{n}$.

For each of the three types of $\mathcal{E}$, we use group-theoretic properties of $\mathcal{E}$ plus ramification data of $K^{\prime} / K_{0}$ to bound (4) from below for large $n$, and then handle the remaining cases individually. Note that among any four consecutive integers $\geq 2$, exactly one of them is prime to 6 , another one is odd, and the other two are even. Recall the notation $d(e)$ from Definition 1 and we see that for $n \geq 6$,

$$
-2+\sum_{j=0}^{3}\left(1-\frac{1}{d(n-j)}\right) \geq-2+4-\frac{1}{2}-\frac{1}{2}-\frac{1}{3}-\frac{1}{5}=\frac{7}{15} .
$$

We will also make repeated use of the following remark. For the rest of this section we will take

$$
V=\{3-n, 2-n, 1-n,-n\},
$$

so if $n \geq 6$ then Lemma 15 implies that the inertia group of any $\nu \in V$ is generated by a single cycle, which will allow us to use Lemma [6 in conjunction with (4).

\section{Case: imprimitive subgroups $S_{j} \prec S_{n / j}$}

First, suppose $n \geq 7$. Since $n-3>n / 2, S_{j} 2 S_{n / j}$ does not contain any $(n-\mu)$-cycle for $0 \leq \mu \leq 3$. That means $c_{1}(\nu)=0$ for every $\nu \in V$. Recall (30) and (4) becomes

$$
g\left(X_{E}\right) \geq 1+\frac{7}{30}\left[S_{n}: S_{j}\left(S_{n / j}\right]>1\right.
$$

Next, suppose $n=6$. The same reasoning as above shows that $c_{1}(\nu)=0$ if $\nu \leq 2-n$, and if $j=2$, then $c_{1}(3-n)=0$ as well. So as before $g\left(X_{E}\right)>1$ if $\mathcal{E} \simeq S_{2} 2 S_{3}$. It remains to consider the case $\mathcal{E} \simeq S_{3} 2 S_{2} \simeq\left(S_{3} \times S_{3}\right) \rtimes \mathbf{Z} / 2$. A representative of the non-trivial coset of $S_{3} \times S_{3}$ in $\left(S_{3} \times S_{3}\right) \rtimes \mathbf{Z} / 2$ (as a subgroup of $S_{6}$ ) is $(14)(25)(36)$; from this we check that elements in this non-trivial coset all have even order. Thus the order 3 elements in $\left(S_{3} \times S_{3}\right) \rtimes \mathbf{Z} / 2$ are all contained in $S_{3} \times S_{3}$. The latter has a unique Sylow 3-subgroup, namely $\mathbf{Z} / 3 \times \mathbf{Z} / 3$, so $\mathcal{E}$ has four distinct $\mathbf{Z} / 3$-subgroups, whence (10) gives $c_{1}(-3)=\frac{8}{\# S_{3} S_{2}} 3 \cdot 3$ ! $=2$. Thus

$$
g\left(X_{E}\right) \geq 1+\frac{6 ! / 72}{2} \frac{7}{15}-\frac{1}{2} \cdot 2 \cdot\left(1-\frac{1}{3}\right)>1
$$

as desired.

Case: intransitive subgroups $S_{n, j}$ with $1 \leq j<n / 2$

For $j>3, S_{n, j}$ contains no cycle of length $\geq n-3$, so $c_{1}(\nu)=0$ for every $\nu \in V$. Thus (414) gives $g\left(X_{E}\right)>1$.

\footnotetext{
${ }^{1}$ i.e. the stabilizer of a partition of $n$ letters into $n / j$ disjoint subsets of equal size
} 
Next, suppose $j=3$, so that we can take $n \geq 7$. Then $c_{1}(\nu)=0$ for $|\nu|>n-3$, and (11) gives $c_{1}(3-n)<6(n-3)$. Thus (4) becomes

$$
g\left(X_{E}\right) \geq 1+\frac{7}{30} \frac{n !}{3 !(n-3) !}-\frac{6 n-19}{2}\left(1-\frac{1}{d(n-3)}\right),
$$

which is easily seen to be $>1$ for $n \geq 7$ (for $n \geq 8$, use the trivial bound $d(n-3) \leq n-3$ ).

Now, take $j=2$. Since $n \geq 6$, the only cycles of order $n-2$ and $n-3$ in $S_{n, 2}=S_{2} \times S_{n-2}$ come from the cycles in $S_{n-2}$ of such order. There are $(n-3) !$ and $(n-2)(n-4)$ ! of them, respectively, so by (10),

$$
c_{1}(n-2)=1 \text { and } c_{1}(n-3)=3,
$$

whence (4) plus (30) gives

$$
g\left(X_{E}\right) \geq 1+\frac{7}{30} \frac{n(n-1)}{2}-\frac{1}{2}\left(1-\frac{1}{d(n-2)}\right)-\frac{3}{2}\left(1-\frac{1}{d(n-3)}\right) .
$$

This is $>1$ for $n \geq 5$, so we are done.

Finally, consider the case $j=1$. Then $X_{E}$ is simply the curve $X_{1}$, which we saw right before the statement of Lemma [10 is simply the curve $\mathcal{L}_{n}$ defined by $L_{n}^{(t)}(x)$. By Proposition 4. this curve has geometric genus $>1$ if $n \geq 6$, so we are done.

Case: primitive subgroups

Let $\mathcal{E} \subset S_{n}$ be a primitive subgroup other than $A_{n}$. By Bochert's theorem [7, p. 79],

$$
\left[S_{n}: \mathcal{E}\right] \geq\left[\frac{n+1}{2}\right] !
$$

Using (11) together with the trivial estimate $1-\frac{1}{d\left(e_{\nu}\right)} \leq 1-\frac{1}{n}$, (41) becomes

$$
\begin{aligned}
g\left(X_{E}\right) & \left.\geq 1+\frac{7}{30}\left[S_{n}: \mathcal{E}\right]-\frac{1}{2}\left(1-\frac{1}{n}\right)((n-1)+(n-2)+(2 n-5))+(6 n-19)\right) \\
& \geq 1+\frac{7}{30}\left[\frac{n+1}{2}\right] !-\left(1-\frac{1}{n}\right) \frac{10 n-27}{2} \\
& \geq 1+\frac{7 \sqrt{\pi n}}{30}\left(\frac{n}{2 e}\right)^{n / 2}-\left(1-\frac{1}{n}\right) \frac{10 n-27}{2} \quad \text { Stirling formula [1, p. 24]. }
\end{aligned}
$$

From (32) we get that $g\left(X_{E}\right)>1$ if $n \geq 15$. Using the sharper form (31), we see that in fact $g\left(X_{E}\right)>1$ if $n \geq 11$. For $n=9,10$, if we use the original inequality (4), we also obtain $g\left(X_{E}\right)>1$. To handle the remaining values of $n$, i.e. $6,7,8$, we make use of classification of primitive groups of small degree [2].

$n=8 \quad S_{8}$ has two maximal primitive subgroups other than $A_{7}$, namely $P G L\left(2, \mathbf{F}_{7}\right)$ and $2^{3} \cdot P S L_{2}\left(\mathbf{F}_{7}\right)$ (a group with normal subgroup $(\mathbf{Z} / 2)^{3}$ and with quotient $P S L_{2}\left(\mathbf{F}_{7}\right)$ ). In particular, both groups contain no element of order 5 , so the $c_{1}$-term in (4) corresponding to the branched point $\nu=-5$ is zero. For the group $P G L_{2}\left(\mathbf{F}_{7}\right)$, (4) then becomes

$$
1+\frac{7}{30} \frac{8 !}{336}-\frac{1}{2}\left(7\left(1-\frac{1}{2}\right)+6\left(1-\frac{1}{7}\right)+11\left(1-\frac{1}{2}\right)\right)>1 .
$$

To handle the group $2^{3} \cdot P S L_{2}\left(\mathbf{F}_{7}\right)$ we need to refine our estimate for the $c_{1}(-7)$-term. Sylow theory dictates that $2^{3} \cdot P S L_{2}\left(\mathbf{F}_{7}\right)$ has at most 64 Sylow 7 -subgroups, all of order 7 , 
so $2^{3} \cdot P S L_{2}\left(\mathbf{F}_{7}\right)$ has at most $64 \times 6=384$ elements of order 7 . Substitute this into (10) and we find that $c_{1}(-7) \leq 2$, whence (4) becomes

$$
1+\frac{7}{30} \frac{8 !}{8 \times 168}-\frac{1}{2}\left(7\left(1-\frac{1}{2}\right)+2\left(1-\frac{1}{7}\right)+11\left(1-\frac{1}{2}\right)\right)>1 .
$$

$n=7 \quad S_{7}$ has a unique maximal primitive subgroup other than $A_{7}$, namely $P S L_{2}\left(\mathbf{F}_{7}\right)$. It has 42 elements of order 4 , no element of order 5 , and 48 elements of order 7 , so $c_{1}(-4)=$ $\frac{42}{168} 4 \cdot 6=6, c_{1}(-5)=0$, and $c_{1}(-7)=\frac{48}{168} \cdot 7=2$, whence (41) becomes

$$
g\left(X_{E}\right) \geq 1+\frac{7}{30} \frac{7 !}{168}-\frac{1}{2}\left(2\left(1-\frac{1}{7}\right)+5\left(1-\frac{1}{2}\right)+6\left(1-\frac{1}{2}\right)\right)>1 .
$$

$n=6 \quad S_{6}$ has a unique maximal primitive subgroup other than $A_{6}$, namely $P G L_{2}\left(\mathbf{F}_{5}\right) \simeq$ $S_{5} \simeq S_{6,1}$. For such intransitive groups we already saw that $g\left(X_{E}\right)>1$, so we are done for $n=6$. This completes the proof of Proposition 5

Proof of Proposition [6. We will make extensive use of the Atlas [3] to determine the maximal subgroups of these $A_{n}$, and for the number of conjugacy classes of elements $A_{n}$ and $P S L_{2}\left(\mathbf{F}_{q}\right)$. For the rest of the proof we take $V=\{-n, 1-n, 2-n, 3-n\}$.

$n=9 \quad$ According to the Atlas, the maximal subgroups ${ }^{2}$ of $A_{9}$ are $A_{8}, S_{7}$, plus others of indices $\geq 84$ in $A_{9}$. First, consider those $\mathcal{E}$ of index $\geq 84$ in $A_{9}$. Then $\left[S_{9}: \mathcal{E}\right] \geq 168$, and (41) becomes

$$
\begin{aligned}
g\left(X_{E}\right) & \geq 1+\frac{7}{30} 168-\frac{1}{2}\left(\left(1-\frac{1}{3}\right) c_{1}(-9)+\left(1-\frac{1}{2}\right) c_{1}(-8)+\left(1-\frac{1}{7}\right) c_{1}(-7)+\left(1-\frac{1}{2}\right) c_{1}(-6)\right) \\
& \geq 1+\frac{196}{5}-\frac{1}{2}\left(\frac{2}{3} 8+\frac{1}{2} 7+\frac{6}{7} 13+\frac{1}{2} 35\right)>1
\end{aligned}
$$

which is satisfactory. Next, take $\mathcal{E}=A_{8}$. Then $\left[S_{9}: \mathcal{E}\right]=18$, and $A_{8}$ has no cycles of order 9,8 or 6 , so $c_{1}(-9)=c_{1}(-8)=c_{1}(-6)=0$. There are $8 ! / 7$ elements of order 7 in $A_{8}$, so $c_{1}(-7)=\frac{8 ! / 7}{8 ! / 2} 7 \cdot 2=4$. Thus

$$
g\left(X_{E}\right) \geq 1+\frac{7 \cdot 18}{30}-\frac{4}{2}\left(1-\frac{1}{7}\right)>1 .
$$

Finally, take $\mathcal{E}=S_{7}$. Then $\left[S_{9}: \mathcal{E}\right]=72$ and $S_{7}$ has no element of order 9 or 8 , so

$$
g\left(X_{E}\right) \geq 1+\frac{7 \cdot 72}{30}-\frac{1}{2}\left(\left(1-\frac{1}{7}\right) 13+\left(1-\frac{1}{2}\right) 35\right)>1 \text {. }
$$

This completes the case $n=9$.

$n=8$ The maximal subgroups of $A_{8}$, along with their indices in $A_{8}$, are

$$
\left(A_{7}, 8\right) ; \quad\left(\left(2^{3}: P S L_{2}\left(\mathbf{F}_{7}\right)\right), 15\right) ; \quad\left(S_{6}, 28\right) ; \quad\left(2^{4}:\left(S_{3} \times S_{3}\right), 35\right) ; \quad\left(\left(A_{5} \times 3\right): 2,56\right) \text {. }
$$

From (11) we get the standard estimates

$$
c_{1}(-8)<8, \quad c_{1}(-7)<7, \quad c_{1}(-6)<6 \cdot 2 \text { !. }
$$

\footnotetext{
2 in what follows we will consider the isomorphism classes, and not conjugacy classes, of maximal subgroups of these $A_{n}$. For the purpose of computing $g\left(X_{E}\right)$ this is sufficient.
} 
The case $\mathcal{E}=2^{3}: P S L_{2}\left(\mathbf{F}_{7}\right)$ has already been dealt with in the course of proving Prop. 5. For $\mathcal{E}=2^{4}:\left(S_{3} \times S_{3}\right)$, it has no element of order 5 or 7 , whence $c_{1}(-5)=c_{1}(-7)=0$. We have $\left[S_{n}: \mathcal{E}\right]=70$, so (41) becomes

$$
g\left(X_{E}\right) \geq 1+\frac{7}{30} 70-\frac{1}{2}\left(\left(1-\frac{1}{2}\right) 8+\left(1-\frac{1}{2}\right) 12\right)>1 .
$$

Next, take $\mathcal{E}=\left(A_{5} \times 3\right)$ : 2, i.e. a split extension with kernel $A_{5} \times \mathbf{Z} / 3$ and quotient $\mathbf{Z} / 2$. The order 5 elements in $\mathcal{E}$ are all in $A_{5} \times \mathbf{Z} / 3$, and hence there are 4 ! of them. Thus (10) gives $c_{1}(-5)=\frac{4 !}{360} 5 \cdot 3 !=2$. Also, $\mathcal{E}$ has no element of order 7 , so $c_{1}(-7)=0$. Thus (111) becomes

$$
g\left(X_{E}\right) \geq 1+\frac{7}{30} 112-\frac{1}{2}\left(\left(1-\frac{1}{2}\right) 8+\left(1-\frac{1}{2}\right) 6+\left(1-\frac{1}{5}\right) 2\right)>1 .
$$

For $\mathcal{E}=S_{6}$, again it has no order 7 elements so $c_{1}(-7)=0$. It has $6 ! / 5$ order 5 elements, so $c_{1}(-5)=\frac{6 ! / 5}{6 !} 5 \cdot 3 !=6$. Thus (41) becomes

$$
1+\frac{7}{30} 56-\frac{1}{2}\left(\left(1-\frac{1}{2}\right) 8+\left(1-\frac{1}{2}\right) 6+\left(1-\frac{1}{5}\right) 6\right)>1
$$

Now take $\mathcal{E}=A_{7}$. There are no cycles of length 6 or 8 in $A_{7}$, so $c_{1}(-8)=c_{1}(-6)=0$. There are 6 ! order 7 elements and $7 ! /\left(5 \cdot 2\right.$ !) order 5 elements in $A_{7}$, so $c_{1}(-7)=c_{1}(-5)=1$. Thus

$$
g\left(X_{E}\right) \geq 1+\frac{7}{30} 8-\frac{1}{2}\left(\left(1-\frac{1}{7}\right)+\left(1-\frac{1}{5}\right)\right)>1 \text {. }
$$

$n=7$ The maximal subgroups of $A_{7}$, along with their indices in $A_{7}$, are

$$
\left(A_{6}, 7\right) ; \quad\left(P S L_{2}\left(\mathbf{F}_{7}\right), 15\right) ; \quad\left(S_{5}, 21\right) ; \quad\left(\left(A_{4} \times 3\right): 2,35\right) .
$$

Note that (11) gives the following estimates

$$
c_{1}(-7)<7, \quad c_{1}(-6)<6, \quad c_{1}(-5)<5 \cdot 2, \quad c_{1}(-4)<4 \cdot 6 .
$$

First, take $\mathcal{E}=\left(A_{4} \times 3\right): 2$. Then $\mathcal{E}$ has no element of order 7 or 5 , so $c_{1}(-7)=c_{1}(-5)=0$. Thus (41) becomes

$$
g\left(X_{E}\right) \geq 1+\frac{7}{30} 70-\frac{1}{2}\left(\left(1-\frac{1}{2}\right) 5+\left(1-\frac{1}{2}\right) 23\right)>1 .
$$

Next, take $\mathcal{E}=S_{5} \subset A_{7}$. Then it has no cycles of order 7 or 6 , so $c_{1}(-7)=c_{1}(-6)=0$. It has 4 ! elements of order 5 , and 5 !/4 elements of order 4 . Thus $c_{1}(-5)=2$ and $c_{1}(-4)=6$. Thus

$$
g\left(X_{E}\right) \geq 1+\frac{7}{30} 42-\frac{1}{2}\left(\left(1-\frac{1}{5}\right) 2+\left(1-\frac{1}{2}\right) 6\right)>1
$$

Now, take $\mathcal{E}=A_{6} \subset A_{7}$. It has no order 7 elements and no cycles of order 6 or 4 . It has $6 ! / 5$ order 5 elements, so $c_{1}(-5)=2$. Thus

$$
g\left(X_{E}\right) \geq 1+\frac{7}{30} 14-\frac{1}{2}\left(1-\frac{1}{5}\right) 2>1 .
$$

Finally, take $\mathcal{E}=P S L_{2}\left(\mathbf{F}_{7}\right)$. It has 42 elements of order 4 , none of order 5 or 6 , and 48 elements of order 7 . Thus $c_{1}(-4)=\frac{42}{168} 4 \cdot 3 !=6, c_{1}(-5)=c_{1}(-6)=0, c_{1}(-7)=\frac{48}{168} 7=2$. Then

$$
g\left(X_{E}\right) \geq 1+\frac{7}{30} 30-\frac{1}{2}\left(\underset{22}{\left(1-\frac{1}{7}\right)} 2+\left(1-\frac{1}{2}\right) 6\right)>1 .
$$


$n=6$ The maximal subgroups of $A_{6}$, along with their indices in $A_{6}$, are

$$
\left(A_{5}, 6\right) ;((\mathbf{Z} / 3 \times \mathbf{Z} / 3) \rtimes \mathbf{Z} / 4,10) ;\left(S_{4}, 15\right) .
$$

First, take $\mathcal{E}=S_{4}$. It has six elements of order 4 , eight of order 3 , and none of order 5 or 6 . Thus $c_{1}(-4)=2, c_{1}(-3)=6, c_{1}(-6)=c_{1}(-5)=0$, whence $g\left(X_{E}\right)>1$.

Next, take $\mathcal{E}=A_{5}$. It has twenty-four elements of order 5 , twenty elements of order 3 , and none of order 6 or 4 . Thus $c_{1}(-5)=1, c_{1}(-3)=3, c_{1}(-6)=c_{1}(-4)=0$, whence $g\left(X_{E}\right)>1$.

Finally, take $\mathcal{E}=(\mathbf{Z} / 3 \times \mathbf{Z} / 3) \rtimes \mathbf{Z} / 4$. Then $c_{1}(-5)=0$. There are 8 elements of order 3 , and hence $\leq 27$ elements of order 4 . Thus $c_{1}(-3)=4$ and $c_{1}(-4) \leq 6$. It follows that $g\left(X_{E}\right)>1$. This completes the proof of Proposition [6.

\subsection{Proof of Theorem 1,}

STEP I. First, we treat the case $n=5$ using an argument specific to quintics. A separable quintic over $K$ (not necessarily irreducible) has a solvable Galois group if and only if its resolvent sextic has a root in $K\left[8\right.$. Compute the resolvent sextic of $L_{5}^{(t)}(x)$ using the formula in [8] and set it to $(x-10 A)\left(x^{5}+c_{1} x^{4}+\cdots+c_{5}\right)$, obtaining six equations in $t, A, c_{1}, \ldots, c_{5}$. Eliminate $c_{1}, \ldots, c_{5}$ from the six equations using MAPLE and we arrive at a single equation in $t$ and $A$ :

$$
\begin{aligned}
A^{6} & +\left(-12 t^{2}-24 t\right) A^{5}+\left(120 t^{2}+60 t^{3}\right) A^{4} \\
& +\left(720 t^{3}+2120 t^{4}+1600 t^{5}+360 t^{6}\right) A^{3} \\
& +\left(-5040 t^{4}-11580 t^{6}-4200 t^{7}-540 t^{8}-13200 t^{5}\right) A^{2} \\
& +\left(10368 t^{4}+39744 t^{5}+48864 t^{6}+14448 t^{7}-12480 t^{8}-9360 t^{9}-1728 t^{10}\right) A \\
& -3\left(5832 t^{5}+26892 t^{6}+50814 t^{7}+50645 t^{8}+28406 t^{9}+8735 t^{10}+1278 t^{11}+54 t^{12}\right) .
\end{aligned}
$$

Using the Algcurves package in MAPle, we find that this equation is absolutely irreducible and defines a plane curve with geometric genus 3. Thanks to Faltings, that means $L_{5}^{(\alpha)}(x)$ is $K$-irreducible and is not solvable for $\alpha \in$ af $K$. This completes the proof for the case $n=5$. From now on, assume that $n \geq 6$.

STEP II. Given a number field $K$, we claim that if there exists one $\beta \in K$ for which $L_{n}^{(\beta)}(x)$ has $S_{n}$-Galois group over $K_{0}$, then Theorem 1 holds for this $K$.

By (25), the discriminant of $L_{n}^{(t)}(x)$ is not constant. Since $n \geq 5$, Lemma 8 applies so that the existence of this one $\beta$ yields the necessary hypotheses on $K^{\prime} / K_{0}$. For $n \geq 10$, the genus of the fixed field of every proper maximal subgroup of $G_{\lambda}$ is greater than 1 (Proposition [5] and Lemma 14). By Riemann-Hurwitz, since $K$ has characteristic $0, g\left(X_{E}\right) \leq g\left(X_{E^{\prime}}\right)$ whenever $E \subset E^{\prime}$. Thus, for $n \geq 10$, every non-trivial intermediate subfield of $K^{\prime} / K_{0}$ has genus greater than 1. For degrees $n=6,7,8,9$, we have shown, (a) that proper maximal subgroups of $A_{n}$ and $S_{n}$ have genus greater than one (Propositions 5 and [6), and (b) over the quadratic subfield of $K_{0}$ in $K^{\prime}$, the polynomials $\Lambda_{j}$ are all irreducible (Proposition (3). Thus, for all $n \geq 6$, the hypotheses of Theorem 3 are satisfied. 
We therefore obtain the first part of Theorem 1(a) for $n \geq 7$. By Lemma 14, if $n \geq 10$ (resp. $n \geq 6$ ) then the set of $t \in K$ corresponding to even Galois groups are parameterized by a curve of geometric genus $\geq 2$ (resp. $\geq 1$ ). The rest of Theorem 1 for $n \geq 7$ now follows.

For $n=6$, the argument for Theorem 3 only shows that the degree of the splitting field of all but finitely many $L_{n}^{(\alpha)}(x)$ over $K$ is divisible by $\operatorname{LCM}\left(\left(\begin{array}{l}6 \\ 2\end{array}\right),\left(\begin{array}{l}6 \\ 3\end{array}\right)\right)=60$. To improve this we use a different test function. By Lemma 8 (a), the fixed field of $K^{\prime} / K_{0}$ by $S_{3} \times\{1\} \subset S_{6,3}$ corresponds to a smooth projective curve $X_{3,0}$ plus a $K$-morphism $\xi_{3,0}: X_{3,0} \rightarrow \mathbf{P}_{K}^{1}$. Write $\Lambda_{3,0}(x, t)=0$ for the corresponding birational plane curve. The same argument as in Lemma 11] shows that the roots of $\Lambda_{3,0}(t)$ over $K_{0}$ are in bijective correspondence with triples of roots of $L_{6}^{(t)}(x)$ over $K_{0}$. Argue as in Proposition 3 and we see that $\Lambda_{3,0}(x, t)$ is irreducible over the fixed field of $K^{\prime} / K_{0}$ by $A_{6}$. The discussion in subsection 6.2 is now applicable, and we see that for $\alpha \in_{\text {af }} K$, the degree of the splitting field of $L_{n}^{(\alpha)}(x)$ over $K$ is divisible by $\operatorname{deg} \xi_{3,0}=\left[S_{6}: S_{3} \times\{1\}\right]=120$. By the classification of transitive subgroups of $S_{6}$ [7, p. 60], we are done.

STEP III. Schur [26] showed that $L_{n}^{(0)}(x)$ is Q-irreducible and has $S_{n}$ Galois group. That means $L_{n}^{(t)}(x)=0$ has $S_{n}$ Galois group over $\mathbf{Q}(t)$. Apply Step II and we get Theorem 1 for $K=\mathbf{Q}$. In particular, $\lambda(x, \alpha)$ has $S_{n}$ Galois group over $\mathbf{Q}$ for all but finitely many $\alpha \in \mathbf{Z}$. From (25) we see that, for any finite set of primes $\Sigma$, infinitely many of these $S_{n}$-extensions of $\mathbf{Q}$ must be ramified outside $\Sigma$. There are only finitely many number fields of bounded degree which are unramified outside $\Sigma$, so for any fixed number field $K$, there exist infinitely

many $\alpha^{\prime} \in \mathbf{Q}$ so that any root of $L_{n}^{\left(\alpha^{\prime}\right)}(x)$ defines a degree $n$ extension $L_{\alpha^{\prime}} / \mathbf{Q}$ with $S_{n}$-Galois closure and is ramified at a prime which is unramified in $K / \mathbf{Q}$. Since $S_{n}$ has no subgroup of index $<n$, that means $L_{\alpha^{\prime}} \cap K=\mathrm{Q}$, whence $L_{n}^{\left(\alpha^{\prime}\right)}(x)$ also has $S_{n}$ Galois group over $K$. Apply Step II with $\beta=\alpha^{\prime}$ and we are done.

\section{Simple COVERS}

Let $Y$ be a smooth projective curve defined over a number field $K$, and let $\pi: Y \rightarrow \mathbf{P}_{K}^{1}$ be a $K$-morphism of degree $n$. We say that $\pi$ is a simple cover if the fiber above every point in $\mathbf{P}_{K}^{1}$ contains at least $n-1$ distinct points. In other words, every branch point of $\pi$ is simple of index 2. By [11, top of p. 549], the (geometric) Galois group of a simple $n$-cover is precisely $S_{n}$. Say $Y$ has genus $g$; then the Riemann-Hurwitz formula implies that the number of branch points of $\pi$ is exactly

$$
\# B_{\pi}=2 g+2 n-2 .
$$

Over an algebraically closed field, if $n \geq g+1$ then every smooth projective curve of genus $g$ admits a simple cover of degree $n$ [11, Prop. 8.1].

Suppose $\lambda(x, t) \in K[x, t]$ is irreducible over $K_{0}=K(t)$ of degree $n$ and defines a simple cover $K_{1} / K_{0}$ (in the notation of section 21). To simplify the exposition, suppose $K$ is algebraically closed in the splitting field $K^{\prime}$ of $\lambda$ over $K_{0}$. The following example of Müller shows that we cannot expect all but finitely $K$-specializations of $\lambda$ to be $K$-irreducible, let alone having the same Galois group as $\lambda$. Consider the transpositions $g_{1}=(1,2), g_{2}=$ $(2,3), \ldots, g_{n-2}=(n-2, n-1), g_{n-1}=(n-1, n), g_{n}=(n-1, n), g_{n+1}=(n-2, n-$ 
$1), \ldots, g_{2 n-3}=(2,3), g_{2 n-2}=(1,2)$. Note that the product of these $g_{i}$ is 1 , and that they generate $S_{n}$. So by the Riemann existence theorem [32, Cor. 7.3], there exists a degree $n$ branched cover $X_{n} \rightarrow \mathbf{P}_{\bar{K}}^{1}$ with exactly $2(n-1)$ branched points over $\bar{K}$, such that the inertia group of the $i$-th branch point is generated by $g_{i}$. By Riemann-Hurwitz, the cover with this description has geometric genus zero and is a simple cover. So taking a finite extension $L / K$ if necessary, there are infinitely many $L$-rational specializations of this cover with an $L$-linear factor.

This example shows that there is not an analogue of Theorem 1 which holds for all simple covers of sufficiently large degree. But if we start with a simple cover of genus at least 2, then we can reach a similar conclusion, as in Part (b) of the following theorem. Even if we start with a rational or elliptic simple cover, however, Part (a) of the theorem says that all but finitely many specializations are either irreducible or factor as a linear times a degree $n-1$ irreducible factor. We give two proofs of Theorem 4. The first is due to Müller, and uses a classification theorem of Liebeck and Saxl; we thank Müller for suggesting that we include it here, as well as for catching an error in an earlier version of the theorem. The second proof illustrates the usefulness of the interpretation of the curve $X_{j}$ introduced in section 5 as the variety whose $K$-rational points parametrize the $K$-rational degree $j$ factors of $\lambda$.

Theorem 4. Let $\lambda(x, t)$ be an irreducible polynomial over $K(t)$ defining a simple cover $\pi: Y \rightarrow \mathbf{P}_{K}^{1}$ of degree $n \geq 5$ and geometric genus $g=g_{Y} \geq 0$. If $g=0$, assume $n \geq 6$. Then,

(a) For $\alpha \in_{a f} K$, the specialization $\lambda(x, \alpha)$ has a $K$-irreducible factor of degree $\geq n-1$.

(b) If $g_{Y} \geq 2$, then for $\alpha \in_{a f} K$, the specialization $\lambda(x, \alpha)$ is $K$-irreducible.

(c) If $g_{Y} \geq 2$ and $n \geq 7$, for $\alpha \in_{a f} K$, the Galois group of $\lambda(x, \alpha)$ over $K$ is $S_{n}$.

First Proof. Suppose $\mathcal{E}$ is a subgroup of $G_{\lambda}$ with fixed field $E$. The key step is the following claim.

Claim. If $\mathcal{E}$ is a maximal subgroup of $G_{\lambda}$ not conjugate to $S_{n, 1}$, then $g_{E} \geq 2$.

We now give a proof, communicated to us by Müller, of this claim. Suppose $\mathcal{E}$ is a maximal subgroup of $S_{n}$ which is not conjugate to $S_{n, 1}$. Recall that $K^{\prime} / K_{0}$ is the Galois closure of the function field extension $K_{1} / K_{0}$ defined by the simple cover $\pi$. This yields an action of $\operatorname{Gal}\left(K^{\prime} / K_{0}\right) \simeq S_{n}$ on the generic fiber of $\pi_{E}$. By Galois theory, this action, call it $\rho_{E}$, is simply the left-action of $S_{n}$ on the left cosets of $\mathcal{E}$ in $S_{n}$. Since $\mathcal{E} \nsucceq S_{n, 1}$, this action is not the natural degree $n$ action of $S_{n}$. Let $\mu(\mathcal{E})$ be the largest integer $m$ such that every transposition of $S_{n}$ moves at least $m$ points in the $\rho_{E}$-action. Since $\pi_{E}$ is a quotient of the Galois closure of the simple cover $\pi$, the ramification index of $\pi_{E}$ at any maximal ideal $\mathfrak{n}$ of an affine coordinate ring of $X_{E}$ divides 2 (Lemma 7). By definition of $\mu(\mathcal{E})$, there are $\mu(\mathcal{E}) / 2$ $\mathcal{O}_{E}$-primes $\mathfrak{n}$ above $\mathfrak{m}$ with $e(\mathfrak{n} / \mathfrak{m})=2$, thus for any $\mathfrak{m} \in B_{\pi}$, as $\mathfrak{n}$ runs through all maximal ideals of $\mathcal{O}_{E}$ lying above $\mathfrak{m}$, we have

$$
\sum_{\mathfrak{n} / \mathfrak{m}}(e(\mathfrak{n} / \mathfrak{m})-1) f(\mathfrak{n} / \mathfrak{m}) \geq \mu(\mathcal{E}) / 2 .
$$

By Lemma 8 (b), the branch locus of $\pi_{E}$ is exactly $B_{\pi}$. Thus Riemann-Hurwitz gives

$$
2\left(N_{E}-1+g_{E}\right) \underset{25}{\geq} \# B_{\pi} \times \mu(\mathcal{E}) / 2 .
$$


Suppose $g_{E} \leq 1$. Then (35) and (34) together give

$$
\mu(\mathcal{E}) \leq 2 N_{E} /\left(n+g_{Y}-1\right) \leq 2 N_{E} /(n-1)
$$

Recall that $\rho_{E}$ is transitive, and since $\mathcal{E}$ is maximal, [7, Cor. 1.5A] implies that $\rho_{E}$ is primitive as well. By [21, Thm. 6.1], either

- $\mu(\mathcal{E}) \geq N_{E} / 2$, or

- $S_{n}$ contains a normal subgroup isomorphic to $H^{r}$, where $H$ is isomorphic to an alternating group $A_{m}$ for some $m$, or to a simple group of Lie type over $\mathbf{Z} / 2$.

The first option plus (36) implies that $n \leq 4$ for $g_{Y} \geq 1$ and $n \leq 5$ for $g_{Y}=0$, and we are done. Since $n \geq 5$, for the second option we must have $m=n, r=1$ and $H \simeq A_{n}$. Furthermore, [21, Thm. 6.1] says that, in this case, $\rho_{E}$ is in fact the action of $S_{n}$ on the set of $j$-subsets of $\{1, \ldots, n\}$ for some $j \in[1, n / 2]$, whence $N_{E}=\left(\begin{array}{c}n \\ j\end{array}\right)$, and $\mu(\mathcal{E})=2\left(\begin{array}{c}n-2 \\ j-1\end{array}\right)$. Recall (36) and we get

$$
2\left(\begin{array}{c}
n-2 \\
j-1
\end{array}\right) \leq \frac{2}{n+g_{Y}-1}\left(\begin{array}{c}
n \\
j
\end{array}\right) .
$$

This inequality simplifies to $j(n-j) \leq n(n-1) /(n+g-1)$. Since $n \geq 5$, this is only possible if $g_{Y} \leq 1$ and $j=1$. Thus, $g_{E} \geq 2$ for all maximal subgroups $\mathcal{E}$ of $G_{\lambda}$ not conjugate to $S_{n, 1}$.

Parts (b) and (c) follow immediately from the claim. Indeed, the hypothesis there, namely $g_{K_{1}}>1$, ensures that the genus of every minimal subfield of $K^{\prime} / K_{0}$ is at least 1 . Now we can apply Proposition 1 and Theorem 3 to complete the proof. For part (a), it remains only to combine the claim with Proposition 2

Second Proof. Now we give a slightly different approach which is independent of LiebeckSaxl. Fix $j \in[1, n / 2]$ and suppose $\lambda(x, \alpha)$ has a $K$-rational degree $j$ factor for infinitely many $\alpha \in K$. Then Proposition 2 implies that $g\left(X_{j}\right) \leq 1$. But the function field of $X_{j}$ is the fixed field $E=K_{j}$ of $\mathcal{E}=S_{n, j}$, so by Lemma 11] (recall the notation introduced at the beginning of subsection [5.2), we have reduced again to the case where $\rho_{E}$ is the action of $G_{\lambda}$ on the set of $j$-subsets of $\Sigma=\left\{\lambda_{1}, \ldots, \lambda_{n}\right\}$. Repeat the argument arising from (37) and we get $g_{Y} \leq 1$ and $j=1$, from which Parts (a) and (b) of the Theorem follow. To prove (c), assuming now that $g_{Y} \geq 2$, and $n \geq 7$, we have already seen that the fixed field of the intransitive maximal subgroups $S_{n, j}$ have genus at least 2. Now we consider a transitive maximal subgroup $\mathcal{E}$ of $G_{\lambda}$. By [30, Lem. 4.4.4], the only transitive subgroup of $S_{n}$ that contains a 2-cycle is $S_{n}$, so we may assume $\mathcal{E}$ has no transpositions. Recalling that $\# B_{\pi}=2 g+2 n-2$, (44) and (10) combine to give $g\left(X_{E}\right)>1$ in this case as well. Now we can apply Theorem 3 to conclude the proof.

Remark 7. The argument above plus Theorem 3 shows that if $g_{Y} \geq 2$ then the Galois group of $\lambda(x, \alpha)$ has order divisible by 60 (if $n=6$ ) and by 20 (if $n=5$ ) for $\alpha \in_{\text {af }} K$. We do not know if the Galois groups are in fact $S_{6}$ and $S_{5}$, respectively, for $\alpha \in_{\text {af }} K$.

Acknowledgments. We are grateful to Professor Müller for pointing out an error in the final section of an earlier draft, and for showing us the first proof of Theorem 4. We would also like to thank Professors Boston, Cox, Gunnells and Markman for useful discussions. 


\section{REFERENCES}

[1] E. Artin, The gamma function. Holt, Rinehart and Winston, 1964.

[2] G. Butler and J. McKay, The transitive subgroups of degree up to eleven. Comm. in algebra 11 (1983) 863-911.

[3] J. H. Conway et al., Atlas of finite groups : maximal subgroups and ordinary characters for simple groups. Oxford, 1985.

[4] D. Cox, Primes of the form $x^{2}+n y^{2}$. Wiley, 1989.

[5] C. J. Cummins and S. Pauli, Congruence subgroups of $P S L(2, \mathbf{Z})$ of genus less than or equal to 24. Exper. Math. 12 (2003) 243-255.

[6] P. Dèbes and M. D. Fried, Integral specialization of families of rational functions. Pacific J. Math. 190 (1999) 45-85.

[7] J. D. Dixon and B. Mortimer, Permutation groups. Springer-Verlag, 1996.

[8] D. Dummit, Solving solvable quintics. Math. Comp. 57 (1991) 387-401.

[9] W. Feit, $\tilde{A}_{5}$ and $\tilde{A}_{7}$ are Galois groups over number fields. J. Algebra 104 (1986) 231-260.

[10] M. Fried, On Hilbert's irreducibility theorem. J. Number Theory 6 (1974) 211-231.

[11] W. Fulton, Hurwitz schemes and irreducibility of moduli of algebraic curves. Ann. Math. 90 (1969) $542-575$.

[12] M. Filaseta and O. Trifonov, The irreducibility of the Bessel polynomials. J. Reine Angew. Math. 550 (2002) 125-140.

[13] M. Filaseta and T. Y. Lam, On the irreducibility of the generalized Laguerre polynomials. Acta Arith. 105 (2002) 177-182.

[14] R. Gow, Some generalized Laguerre polynomials whose Galois groups are the alternating groups. J. Number Theory 31 (1989) 201-207.

[15] F. Hajir, Some $\tilde{A}_{n}$-extensions obtained from generalized Laguerre polynomials. J. Number Theory 50 (1995) 206-212.

[16] F. Hajir, Algebraic properties of a family of generalized Laguerre polynomials, preprint, 17pp.

[17] M. Hall, The theory of groups. Macmillan, 1959.

[18] J. B. Dennin, Jr., The genus of subfields of K(n). Proc. A.M.S. 51 (1975) 282-288.

[19] M. I. Knopp and M. Newman, Congruence subgroups of positive genus of the modular group. Ill. J. Math. 9 (1965) $577-583$.

[20] S. Lang, Elliptic functions, 2nd ed. Springer-Verlag, 1987.

[21] M. W. Liebeck and J. Saxl, Minimal degrees of primitive permutation groups, with an application to monodromy groups of covers of Riemann surfaces. Proc. London Math. Soc. 63 (1991) 266-314.

[22] A. M. Macbeath, Extensions of the rationals with Galois group $\operatorname{PGL}\left(2, \mathbf{Z}_{n}\right)$. Bull. London Math. Soc. 1 (1969) 332-338.

[23] P. Müller, Finiteness results for Hilbert's irreducibility theorem. Ann. Inst. Fourier 52 (2002) 983-1015.

[24] M. Rosen, Number theory in function fields. Springer-Verlag, 2002.

[25] I. Schur, Einige Sätze über Primzahlen mit Anwendungen auf Irreduzibilitätsfragen. II. Sitzungsberichte der Berliner Akademie (1929) 370-391.

[26] I. Schur, Gleichungen ohne Affekt. Sitzungsberichte der Berliner Akademie (1930) 443-449.

[27] I. Schur, Affektlose Gleichungen in der Theorie der Laguerreschen und Hermiteschen Polynome. J. reine und angew. Math. 165 (1931) 52-58.

[28] E. Sell, On a family of generalized Laguerre polynomials, To appear in J. Number Theory, 13pp.

[29] J. P. Serre, Lectures on the Mordell-Weil theorem, 2nd ed. Vieweg, 1990.

[30] J. P. Serre, Topics in Galois theory. Jones and Bartlett Publ., 1992.

[31] J. H. Silverman, The arithmetic of elliptic curves. Springer-Verlag, 1986.

[32] H. Volklein, Groups as Galois groups : an introduction. Cambridge University Press, 1996.

E-mail address: hajir@math.umass.edu

Department of Mathematics \& Statistics, University of Massachusetts. Amherst, Ma 01003-9318 USA

E-mail address: siman@math.umass.edu 
Department of Mathematics \& Statistics, University of Massachusetts. Amherst, Ma 01003-9318 USA 